\title{
Robust Compound Control of Dynamic Bipedal Robots
}

\author{
Jaime Arcos-Legarda ${ }^{\mathrm{a}}$, John Cortes-Romero ${ }^{\mathrm{b}}$, Andres Tovar $^{\mathrm{c}}$ \\ ${ }^{a}$ Associate Professor \\ Department of Mechatronic Engineering \\ Universidad de San Buenaventura \\ Bogotá, Colombia \\ Email:wjarcosl@unal.edu.co \\ ${ }^{b}$ Associate Professor \\ Department of Electrical and Electronic Engineering \\ Universidad Nacional de Colombia \\ Bogotá, Colombia \\ Email: jacortesr@unal.edu.co \\ ${ }^{c}$ Associate Professor \\ Department of Mechanical and Energy Engineering \\ Indiana University-Purdue University Indianapolis \\ Indianapolis, Indiana, USA \\ Email: tovara@iupui.edu
}

\begin{abstract}
This paper presents a robust compound control strategy to produce a stable gait in dynamic bipedal robots under random perturbations. The proposed control strategy consists of two interactive loops: an adaptive trajectory generator and a robust trajectory tracking controller. The adaptive trajectory generator produces references for the robot controlled joints without a-priori knowledge of the terrain features and minimizes the effects of disturbances and model uncertainties during the gait, particularly during the support-leg exchange. The trajectory tracking controller is a non-switching robust multivariable generalized proportional integral (GPI) controller. The GPI controller rejects external disturbances and uncertainties faced by the robot during the swing walking phase. The proposed control strategy was evaluated on the numerical model of a five-link planar bipedal robot with one degree of under-actuation, four actuators, and point feet. The results showed robust performance and stability under external disturbances and model parameter uncertainties on uneven terrain with uphills and downhills. The stability of the gait was proven through the computation of a Poincaré return map for a hybrid zero dynamics with uncertainties (HZDU) model, which shows con-
\end{abstract}

Preprint submitted to Mechatronics

October 7, 2019

This is the author's manuscript of the article published in final edited form as:

Arcos-Legarda, J., Cortes-Romero, J., \& Tovar, A. (2019). Robust compound control of dynamic bipedal robots. Mechatronics, 59, 154-167. https://doi.org/10.1016/j.mechatronics.2019.04.002 
vergence to a bounded neighborhood of a nominal orbital periodic behavior.

Keywords: Dynamic Bipedal Robots, Generalized Proportional Integral Control, Virtual Holonomic Constraints, Hybrid Zero Dynamics.

\section{Introduction}

After more than five decades of research on stability and motion control, bipedal robots have evolved from passive walking mechanisms, e.g., [1], to sophisticated humanoids with the ability to run and evade obstacles, e.g., 2]. Due to their potential application in tasks such as rescue missions [3], space exploration [4], and rehabilitation [5, 6], research on bipedal robot motion remains increasingly active. According to the control strategy, walking robots can be divided into two groups: (1) walkers with static stability and (2) dynamic walkers. Walkers with static stability have achieved robust gaits with skills to interact with complex environments - an outstanding example is Honda R\&D's ASIMO [7]. The control of walkers with static stability is based on the zero moment point (ZMP) principle [8]. The ZMP principle ensures static stability by locating a reaction point inside a supporting polygon defined by the area under the robot's feet. This control strategy provides the robustness needed to reject disturbances generated by unstructured environments and allows them to move in areas built for humans [9]. While effective, the use of the ZMP principle requires flat contact between the feet and the ground during the complete gait cycle, which results in unnatural movements and high energy consumption.

Dynamic walkers have been designed to utilize the potential energy of their body, decrease energy consumption, and attain a natural gait [10, 11]. These robots can be described with hybrid dynamic models composed of continuous dynamic functions connected by discrete reset functions. The continuous dynamic functions simulate the swing phase of the gait and the discrete reset functions simulate the support-leg exchange. The hybrid dynamics produced by the continuous and discrete functions are handled by a compound control strategy that uses two interacting loops: a trajectory generator and a trajectory tracking controller. The trajectory generator consists of a set of off-line reference trajectories satisfying holonomic constraints. These constraints, also referred to as virtual holonomic constraints (VHC), are induced through a feedback control loop [12, 13, 14, 15]. The off-line reference trajectories are the solution of a parameter optimization problem 
consisting of minimizing the mechanical cost of transportation (MCT) satisfying natural kinematic and dynamic constraints of a reduced model, i.e., hybrid zero dynamics (HZD) [16]. Exponentially stable periodic orbits of the HZD correspond to exponentially stabilizable orbits of the full model.

The trajectory tracking controller implements feedback linearization and proportional derivative controllers [16, 17]. Recently, robust nonlinear controllers have been proposed [18, 19, 20, 21, 22, 23, 24, 25, 26]. These controllers can deal with random variations of the terrain through exhaustive trajectory generation strategies and the optimization of trajectory references or VHC [18, 19, 20]. Nonlinearities such as the physical saturation of the actuators have been also addressed through optimal nonlinear control strategies nonholonomic, virtual constraints to generate gait trajectories [21, 22, 27, 26]. Unfortunately, such approaches rely on the accuracy of the robot model. Recently, disturbance observers have been developed to estimate and reject internal and external disturbance. [28, 29]. A limitation of these strategies is the need of an explicit state observer. Benefits in robustness have been also explored with the use of a motorized reaction wheel [30, 31]. However, these current compound control strategies are susceptible to model mismatching and external disturbances in bipedal robots. This is evident during the support-leg exchange event where the reference trajectories and the tracking errors suffer a sudden change. This produces high transitory responses and, ultimately, the instability of the gait. Examples of high transitory responses on physical prototypes can be found in [32, 21]. To alleviate this problem, VHC modifications have been proposed in 2D and 3D bipedal robots [33, 28]. Even though this technique improves gait stability, it requires an event-based controller to select a VHC from a repository of reference trajectories.

This paper introduces a robust compound control strategy for dynamic walkers. The trajectory generator of the proposed strategy uses modified VHC, which include the step-length as one of the control variables. This allows (1) to modify the walking pattern to address internal and external disturbances such as model mismatching, external disturbance forces, and changes in the terrain topography, and (2) to generate trajectories for stable and smooth cyclic walking under reasonable deviations of the nominal states during the support-leg exchange event. The trajectory tracking controller of the proposed strategy is a multivariable robust GPI controller [34. This GPI controller, which is formulated within the scope of an active disturbance rejection control (ADRC) framework [35, 29], assumes that the endogenous and exogenous disturbances can be lumped into a total disturbance signal 
[36, 37, 38]. The GPI controller has been successfully used in a flat-foot bipedal gait exoskeleton [39] and a bipedal robot with static stability [40]. In this work, the GPI controller is further extended to trajectory tracking of underactuated dynamic bipedal robots.

The effectiveness of the robust compound control strategy is demonstrated with the analysis of the total walking stability of a dynamic bipedal robot. The Poincaré section method is used to verify the existence of a bounded neighborhood around a nominal, periodic, and stable orbit in the states of the HZD. The existence of such neighborhood allows to conclude that the full hybrid model also has a neighborhood around a nominal, periodic, and stable orbit, which proves gait stability under bounded model uncertainties and bounded disturbances.

This paper is organized as follows: Section 2 describes the hybrid model of the dynamic bipedal robot. Section 3 presents the multivariable robust GPI control strategy. Section 3.4 analyzes the HZD for the uncertain dynamic bipedal robot. Section 4 shows the results of the total stability test in which the Poincaré return map is used. In Section 5, an adaptive virtual constraint is proposed as trajectory generation strategy; here, a technique to minimize the transitory responses in the support-leg change event is also presented. Section 6 contains the numerical evaluation and physical experimentation of the proposed strategies. Finally, Section 7 summarizes the accomplishments of this work and draws recommendations for future developments.

\section{Dynamic Bipedal Robot Hybrid Model}

The bipedal robot prototype considered in this work is shown in Figure 1 . This robot consists of five rigid links forming a torso, two identical legs, and a hip. Each legs is composed of a thigh and a shin connected by a revolute joint working as a knee. The legs-ends have a point-foot without ankle. The robot's hip is attached to a bar that constraints the gait to a circular path; however, for all practical purposes, it is assumed that the robot follows a straight path and all movements are on the sagittal plane.

The robot has seven degrees of freedom (DOFs) as shown in Figure 1; two thigh angles $h_{r}$ and $h_{l}$ at the right and left side of the hip, two shin angles $k_{r}$ and $k_{l}$ at the right and left knees, one angle at the torso $q_{T}$ with respect to a global fixed frame, and two cartesian coordinates $x$ and $y$ of the standing leg. 
It is assumed that the double support phase of the gait is instantaneous and that the support leg-end in the swing phase has unilateral constraints. In this way, it does not have rebound or slip, its normal reaction force is repulsive, and the (tangential) friction force is inside of a friction cone. The unilateral constraints transform the support leg-end into a pivot and reduces the DOFs from seven to five. Therefore, the robot is designed with only four actuators: two in the hip and two in the knees. The robot has one degree of underactuation.
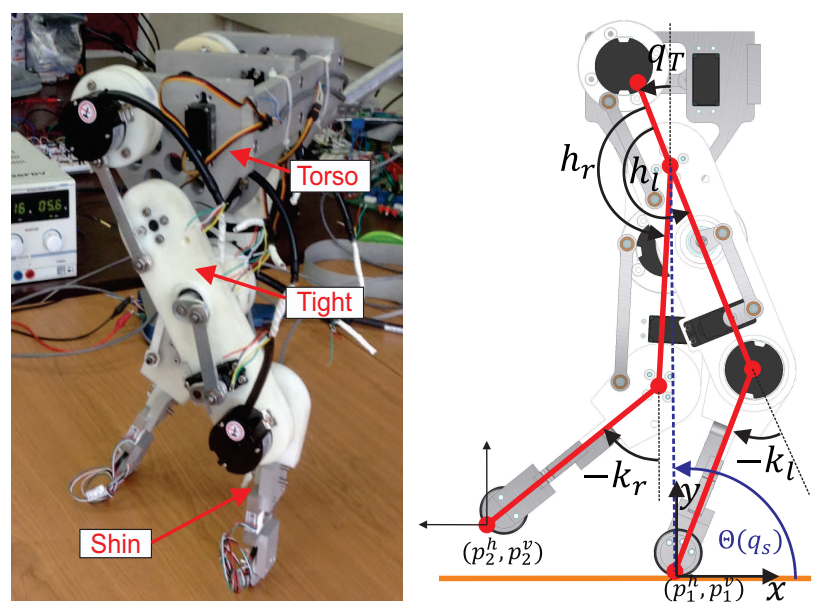

Figure 1: Multibody model of the bipedal robot and robot prototype

In this work, the Lagrange differential equation is used to find a perturbed model of the robot in the swing phase. For detaills about the model construction see: [17, 36, 41]. The resulting Euler-Lagrange equation is

$$
D_{s}\left(q_{s}\right) \ddot{q}_{s}+C_{s}\left(q_{s}, \dot{q}_{s}\right) \dot{q}_{s}+G_{s}\left(q_{s}\right)=B_{s}\left(q_{s}\right) u+\delta\left(q_{s}, \dot{q}_{s}\right)+\zeta
$$

where $q_{s}$ is a vector of the generalized coordinates. The configuration of the vector $q_{s}$ dependents on which leg is in support. This is,

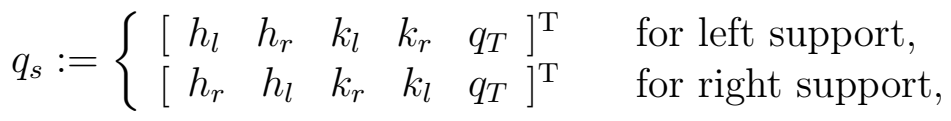

$D_{s}\left(q_{s}\right)$ is the inertial matrix, $C_{s}\left(q_{s}, \dot{q}_{s}\right) \dot{q}_{s}$ is a vector of centripetal and Coriolis effects, $G_{s}\left(q_{s}\right)$ is a vector of torques associated to the gravity, $B_{s}\left(q_{s}\right) u$ is a 
vector of generalized torques, $\delta\left(q_{s}, \dot{q}_{s}\right)$ is an unknown bounded vector of model uncertainties, and $\zeta$ is an unknown bounded vector of external disturbances.

The perturbed model can be expressed in state space by rewriting (1) in the general input affine form

$$
\dot{x}=f(x)+g(x) u+\gamma(x, t),
$$

where $x:=\left[\begin{array}{ll}q_{s}^{\mathrm{T}} & \dot{q}_{s}^{\mathrm{T}}\end{array}\right]^{\mathrm{T}}$ is the state space vector in $\mathbb{R}^{n}$,

$$
\begin{aligned}
f(x) & :=\left[\begin{array}{c}
\dot{q}_{s} \\
D_{s}\left(q_{s}\right)^{-1}\left[-C_{s}\left(q_{s}, \dot{q}_{s}\right) \dot{q}_{s}-G_{s}\left(q_{s}\right)\right]
\end{array}\right], \\
g(x) & :=\left[\begin{array}{c}
0 \\
D_{s}\left(q_{s}\right)^{-1} B_{s}
\end{array}\right], \\
\gamma(x, t) & :=\left[\begin{array}{c}
0 \\
D_{s}\left(q_{s}\right)^{-1}\left[\delta\left(q_{s}, \dot{q}_{s}\right)+\zeta(t)\right]
\end{array}\right] .
\end{aligned}
$$

The support-leg exchange is a discrete event that can be modeled as rigid body collision [42. To find a suitable mathematical description, our work follows the approach proposed by [43, 16]. The connection between the continuous dynamics of the swing leg phases is given by an instantaneous double support phase, which is modeled by a discrete reset function that maps the state variables from the values just before the support-leg exchange to the values after it. In order to consider the uncertainties of the model, a reset function with uncertainties is proposed to describe the discrete dynamics in the support-leg exchange. This is,

$$
x^{+}:=\Delta\left(x^{-}\right)+\gamma_{\Delta},
$$

where $x^{-}$and $x^{+}$are the state variables just before and after the supportleg exchange, respectively. $\Delta\left(x^{-}\right)$is a reset function that under nominal condition maps the states variables before the support-leg exchange to the states after the support-leg exchange. This function is described by

$$
\Delta\left(x^{-}\right):=\left[\begin{array}{c}
\Delta_{q} q_{s}^{-} \\
\Delta_{\dot{q}}\left(q_{s}^{-}\right) \dot{q}_{s}^{-}
\end{array}\right]
$$

where $\Delta_{q}$ represents the angles relabeling in the role change of the legs in the support-leg exchange and $\Delta_{\dot{q}}$ is a function that resets and relabels the angular velocities [17]. Given that $\Delta_{q}$ and $\Delta_{\dot{q}}$ are functions of the robot's 
parameters, the uncertainties in such parameters produce also uncertainties in the state variable after the support-leg exchange. In order to model the effects of such uncertainties, an input-equivalent disturbance $\gamma_{\Delta}$ is added to the reset map function as shown in (7).

The hybrid model considers the continuous model (3) and the discrete reset function (7) as

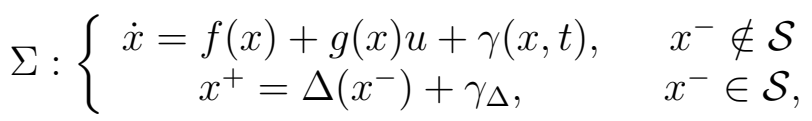

where the switching set is defined as

$$
\mathcal{S}:=\left\{\left[\begin{array}{ll}
q_{s}^{\mathrm{T}} & \dot{q}_{s}^{\mathrm{T}}
\end{array}\right]^{\mathrm{T}} \in \mathbb{R}^{n} \mid p_{2}^{v}\left(q_{s}\right)=d, \dot{p}_{2}^{v}\left(q_{s}, \dot{q}_{s}\right)<0\right\},
$$

where $p_{2}^{v}\left(q_{s}\right)$ is the vertical Cartesian position of the swing leg-end, and $d$ is the terrain height. The terrain height is defined with respect to the support leg-end. In this way, $d=0$ represents a flat terrain, which corresponds to walking on nominal condition, $d>0$ indicates an uphill terrain, and $d<0$ is a downhill terrain. If the terrain is rough (uncertain), then $d$ is random. For control design purpose $d$ is considered constant $d=0$ and the robustness of the control strategy is evaluated in bonded values of $d$ such as

$$
d_{\min } \leq d \leq d_{\max }
$$

where $d_{\text {min }}$ and $d_{\text {max }}$ are the minimum and maximum terrain heights, respectively.

Since the model uncertainties and the external disturbances act collectively attempting against the gait stability, this work develops in Section 3 a robust multivariable control strategy to reject disturbances in the continuous dynamics of the actuated robot joints. On the other hand, Section 5 develops an adaptive trajectory generation strategies to overcome the disturbances in the discrete dynamics and underactuated degrees of freedom.

\section{Robust Multivariable Generalized Proportional Integral (GPI) Controller}

Robust generalized proportional integral controllers are known to efficiently track trajectories and reject input perturbations avoiding the explicit

use of state observers [44]. Instead, a structural reconstruction of the state 
variables is performed through iterated integrations of the inputs, outputs, and the errors, which complete the stable feedback design. As a result, control laws based on state feedback can be implemented without the necessity of asymptotic observers [45]. However, the necessary conditions to apply GPI controllers to underactuated hybrid dynamic systems is still unclear.

In order to define the operation conditions of a GPI controller in a hybrid dynamic system, a robust multivariable GPI controller is designed into four stages. First, a model decomposition is performed to isolate the actuated joints from the underactuated one. Second, a partial feedback linearization is performed to linearize the actuated part of the continuous dynamics. Third, a chain of iterated integral reconstructors is built to form the GPI control structure. Finally, the internal dynamics of the robot is analyzed with the definition of a hybrid zero dynamics with uncertainties.

\subsection{Model decomposition}

In order to apply the GPI controller to the trajectory tracking problem of a dynamic bipedal robot, the continuous model (1) is written in compact form as

$$
D_{s}\left(q_{s}\right) \ddot{q}_{s}+\Omega\left(q_{s}\right)=B_{s}\left(q_{s}\right) u+\tilde{\gamma}_{\xi}\left(q_{s}, \dot{q}_{s}\right)
$$

where

$$
\begin{aligned}
\Omega\left(q_{s}\right) & :=G_{s}\left(q_{s}\right), \\
\tilde{\gamma}_{\xi}\left(q_{s}, \dot{q}_{s}\right) & :=-C_{s}\left(q_{s}, \dot{q}_{s}\right) \dot{q}_{s}+\delta\left(q_{s}, \dot{q}_{s}\right)+\zeta .
\end{aligned}
$$

Now, let us decompose the perturbed model (12) into the actuated and the underactuated parts of the system. This is:

$$
\begin{aligned}
& {\left[\begin{array}{ll}
D_{11}\left(q_{s}\right) & D_{12}\left(q_{s}\right) \\
D_{21}\left(q_{s}\right) & D_{22}\left(q_{s}\right)
\end{array}\right]\left[\begin{array}{c}
\ddot{q}_{b} \\
\ddot{q}_{N}
\end{array}\right]+\left[\begin{array}{l}
\Omega_{1}\left(q_{s}\right) \\
\Omega_{2}\left(q_{s}\right)
\end{array}\right]=\cdots} \\
& \cdots=\left[\begin{array}{c}
B_{1}\left(q_{s}\right) \\
0
\end{array}\right] u+\left[\begin{array}{c}
\tilde{\gamma}_{\xi 1}\left(q_{s}, \dot{q}_{s}\right) \\
\tilde{\gamma}_{\xi 2}\left(q_{s}, \dot{q}_{s}\right)
\end{array}\right] .
\end{aligned}
$$

where $q_{b}$ are the controlled joints and $q_{N}$ is the underactuated DOF. In our robot this corresponds to the torso absolute angle $q_{T}$. Then, the decomposed model representation (15) is used to express the model as a function of the actuated variables as follows:

$$
\ddot{q}_{b}=\kappa\left(q_{s}\right) u+\psi\left(q_{s}\right)+\gamma_{\xi}\left(q_{s}, \dot{q}_{s}\right)
$$


where

$$
\begin{aligned}
\kappa\left(q_{s}\right) & :=\kappa_{1}^{-1} B_{1}\left(q_{s}\right), \\
\gamma_{\xi}\left(q_{s}, \dot{q}_{s}\right) & :=\kappa_{1}^{-1}\left(\tilde{\gamma}_{\xi 1}\left(q_{s}, \dot{q}_{s}\right)-D_{12}\left(q_{s}\right) D_{22}^{-1}\left(q_{s}\right) \tilde{\gamma}_{\xi 2}\left(q_{s}, \dot{q}\right)\right), \\
\psi\left(q_{s}\right) & :=\kappa_{1}^{-1}\left(-\Omega_{1}\left(q_{s}\right)+D_{12}\left(q_{s}\right) D_{22}^{-1}\left(q_{s}\right) \Omega_{2}\left(q_{s}\right)\right) \\
\kappa_{1} & :=\left[D_{11}\left(q_{s}\right)-D_{12}\left(q_{s}\right) D_{22}^{-1}\left(q_{s}\right) D_{21}\left(q_{s}\right)\right] .
\end{aligned}
$$

\subsection{Partial feedback linearization}

The following assumptions are proposed for the lumped disturbance vector $\gamma_{\xi}\left(q_{s}, \dot{q}\right)$ in 16$)$ :

(i) $\gamma_{\xi}\left(q_{s}, \dot{q}_{s}\right)$ contains endogenous and exogenous disturbances,

(ii) $\gamma_{\xi}\left(q_{s}, \dot{q}_{s}\right)$ can be locally approximated, on a time window around the current time, by self-updating time polynomials of $m-1$ order,

(iii) $\gamma_{\xi}\left(q_{s}, \dot{q}_{s}\right)$ and its first $m$ time-derivatives are bounded almost everywhere. In such a way, there exists a finite constant $\varepsilon_{i}$ that satisfies $\sup \left|\gamma_{\xi}^{(i)}\right| \leq \varepsilon_{i}, i=0, \ldots, m$ for all $t \geq 0$ except during the discontinuities of the lumped disturbance signals.

In a self-updating fashion, this allows to reject the disturbance effects through the base of its internal model [44, 46]. In our robot, this is taken as

$$
\frac{d^{m}}{d t^{m}} \gamma_{\xi}^{j}\left(q_{s}, \dot{q}_{s}\right) \approx 0
$$

for $j=1,2, \ldots, k$, and $m=3$.

With the aforementioned assumptions, the following control law is proposed:

$$
u=u_{\xi}^{*}+v_{\xi}
$$

with

$$
\begin{aligned}
& u_{\xi}^{*}=\kappa^{-1}\left(q_{s}\right)\left(-\psi\left(q_{s}\right)\right), \\
& v_{\xi}=\kappa^{-1}\left(q_{s}\right)\left(u_{\mathrm{GPI}}\right) .
\end{aligned}
$$

By applying the control law (18) to the system (16), the tracking control task is transformed into a set of Single-Input and Single-Output (SISO) control 
design problems, instead of a more complex Multiple-Input and MultipleOutput (MIMO) problem.

The effects of the lumped disturbances $\gamma_{\xi}^{j}\left(q_{s}, \dot{q}\right)$ are dynamically coupled and it is not possible to mathematically decouple them. Remarkably, the GPI controller rejects the effect of such coupling. Thus, each SISO problem can be represented by an integrator chain $\ddot{q}_{b_{j}}$ and the lumped disturbance variable $\gamma_{\xi}^{j}\left(q_{s}, \dot{q}\right)$ as

$$
\ddot{q}_{b_{j}}=u_{\mathrm{GPI}}^{j}+\gamma_{\xi}^{j}\left(q_{s}, \dot{q}\right)
$$

where $u_{\mathrm{GPI}}^{j}$ is the control signal for the $j$ th joint produced by the GPI controller.

\subsection{Integral reconstructors}

The GPI controller is developed in the context of output trajectory tracking. It takes into account desired trajectories of the actuated joints. The desired trajectories can be defined by a vector $q_{d_{4 \times 1}}$. The design of $q_{d}$ will be discussed in Section 5. Thus, the output tracking error is governed by

$$
\ddot{e}_{q_{j}}=e_{u^{j}}+\gamma_{\xi}^{j}\left(q_{s}, \dot{q}\right)
$$

where $e_{q_{j}}=q_{b_{j}}(s)-q_{d_{j}}(s)$ is the tracking error vector, $e_{u^{j}}=u_{\mathrm{GPI}}^{j}-u_{\mathrm{GPI}}^{j *}$ is the control signal error and $u_{\mathrm{GPI}}^{j *}=\ddot{q}_{d_{j}}$ is the nominal control signal, so

$$
e_{u^{j}}=u_{\mathrm{GPI}}^{j}-\ddot{q}_{d_{j}} .
$$

The regulation of $(22)$ can be achieved with a state feedback controller as follows:

$$
e_{u^{j}}=-k_{j, n+m} \hat{\dot{e}}_{q_{j}}-k_{j, n+m-1} e_{q_{j}},
$$

where $\hat{\dot{e}}_{q}$ is an estimation of $\dot{e}_{q}$. This can be computed by neglecting the total disturbance terms in (22) and applying the integral reconstructors

$$
\hat{\dot{e}}_{q}=\int_{0}^{t} e_{u}\left(\sigma_{1}\right) d \sigma_{1}
$$

Integrating (22) and replacing in (25), the estimation takes the form

$$
\hat{\dot{e}}_{q}=\dot{e}_{q}(t)-\dot{e}_{q}(0)-\int_{0}^{t} \gamma_{\xi}\left(\sigma_{1}\right) d \sigma_{1} .
$$


The use of the integral reconstructor on the states estimation (26) avoids the need for an asymptotic state observer. However, this integral reconstructor does not consider the initial conditions, which produces a discrepancy between the reconstructed states and their actual values. To compensate this discrepancy, an integral of the tracking errors is included in the feedback controller (24) [46]. The resulting controller can be expressed as

$$
e_{u_{j}}=-k_{j, n+m} \hat{\dot{e}}_{q_{j}}-k_{j, n+m-1} e_{q_{j}}-k_{j, n+m-2} \int_{0}^{t} e_{q_{j}}\left(\sigma_{1}\right) d \sigma_{1} .
$$

The integral of the tracking errors in (27) banishes the estimation error caused by the initial conditions.

In order to increase the robustness of the controller against unmodeled dynamics and the external disturbances, a finite number of iterated integrals of the tracking errors are added to the proposed control (27). The number of iterated integrals is defined by the degree of the derivative in the internal model assumption (17). Hence, $m+1$ iterated integrals are included in the feedback controller of our robot. These take the form

$$
\begin{aligned}
e_{u_{j}}= & -k_{j, n+m} \hat{\dot{e}}_{q_{j}}-k_{j, n+m-1} e_{q_{j}} \ldots \\
& \cdots-k_{j, n+m-2} \int_{0}^{t} e_{q_{j}}\left(\sigma_{1}\right) d \sigma_{1} \cdots \\
& \cdots-k_{j, n+m-3} \int_{0}^{t} \int_{0}^{\sigma_{1}} e_{q_{j}}\left(\sigma_{2}\right) d \sigma_{2} d \sigma_{1} \cdots \\
\vdots & \\
& \cdots-k_{j, 0} \int_{0}^{t} \int_{0}^{\sigma_{1}} \cdots \int_{0}^{\sigma_{m}} e_{q_{j}}\left(\sigma_{m+1}\right) d \sigma_{m+1} \cdots d \sigma_{2} d \sigma_{1} .
\end{aligned}
$$

An explicit expression for the GPI controller can be obtained replacing (25) into (28). Substituting the nominal control signal $u_{\mathrm{GPI} i}^{*}(s)$ and applying Laplace transform yields

$$
u_{\mathrm{GPI} i}(s)=u_{\mathrm{GPI} i}^{*}(s)-\left(\frac{k_{j, m+1} s^{m+1}+\cdots+k_{j, 1} s+k_{j, 0}}{s^{m}\left(s+k_{j, m+n}\right)}\right) e_{q_{j}}(s) .
$$

Substituting (28) and (25) into (22) leads to the dynamic equation that describes the closed-loop behavior. Applying Laplace transform, the closedloop behavior can be expressed as

$$
\begin{array}{r}
\left(s^{m+n+1}+k_{j, m+n} s^{m+n}+\cdots+k_{j, 1} s+k_{j, 0}\right) e_{q_{j}}(s)=\cdots \\
\cdots=s^{m} \gamma_{\xi}^{j}(s)\left(s+k_{j, m+n}\right)+s^{m} k_{j, m+n} \dot{e}_{q}(0) .
\end{array}
$$


Given that the term $k_{j, m+n} \dot{e}_{q}(0)$ is a constant due to the initial conditions, then

$$
s^{m} k_{j, m+n} \dot{e}_{q}(0)=0 .
$$

According to the internal model assumption (17),

$$
s^{m} \gamma_{\xi}^{j}(s)\left(s+k_{j, m+n}\right) \approx 0 .
$$

In order to impose a dominant dynamic on the tracking errors, the designer can select the gains $k_{j, i}$ of the GPI controller. The appropriate selection of these gains results in tracking errors that are ultimately, uniformly, and absolutely bounded [47]. Finally, a dominant characteristic polynomial of the closed-loop system is

$$
\left(s^{m+n+1}+k_{j, m+n} s^{m+n}+\cdots+k_{j, 1} s+k_{j, 0}\right) e_{q_{j}}(s) \approx 0 .
$$

\subsection{Hybrid Zero Dynamics with Uncertainties}

The robust multivariable GPI controller is designed to track a set of four trajectory references $q_{d_{1}}, \ldots, q_{d_{4}}$. An underactuated (not controllable) DOF $q_{5}$, corresponding to the torso absolute angle, produces the internal dynamics that defines the stability of the robot. In order to analyze the stability of the internal dynamics, let us consider that the GPI controller creates a submanifold where the tracking error of the controlled joints converges to a bounded vicinity of zero. Thus, the dynamics of the full hybrid model (9) is constrained by the conditions $e_{q_{j}} \approx 0$ and $\dot{e}_{q_{j}} \approx 0$. These conditions allow to define a hybrid zero dynamics with uncertainties (HZDU) as

$$
\Sigma_{z}: \begin{cases}\dot{z}=f_{z}(z)+\Xi, & z^{-} \notin \mathcal{Z}, \\ z^{+}=\Delta_{z}\left(z^{-}\right)+\Xi_{\Delta}, & z^{-} \in \mathcal{Z} .\end{cases}
$$

where $\mathcal{Z} \subset \mathcal{S}$ and it is defined as

$$
\mathcal{Z}:=\left\{\left[\begin{array}{ll}
q_{s}^{\mathrm{T}} & \dot{q}_{s}^{\mathrm{T}}
\end{array}\right]^{\mathrm{T}} \in \mathcal{S} \mid q_{b}=q_{d}, \dot{q}_{b}=\dot{q}_{d}\right\},
$$

$f_{z}(z)$ and $\Delta_{z}\left(z^{-}\right)$are the zero dynamics and its reset map without disturbances, respectively. The vectors $\Xi$ and $\Xi_{\Delta}$ represent the discrepancy between the nominal hybrid zero dynamics and the internal dynamics under uncertainties and disturbances. The proposed HZDU allows to analyze the full hybrid model stability with the reduced order subsystem (34). Details of its derivation are included in Appendix A. The existence of bounded orbital behaviors in the presence of lumped disturbances is demonstrated in the following section. 


\section{Orbital Gait Stability Analysis}

In order to define the conditions that provide gait stability in the presence of external disturbances and model uncertainties, let us define $\varphi(t)$ as the trajectory that results from the solution of (34). Under the assumption of zero disturbances and zero uncertainties, the gait of the robot is periodic if the trajectory $\varphi(t)$ belongs to a nominal periodic orbit $\mathcal{O}$, such that $\varphi(t+T)=$ $\varphi(t)$, where $T$ is the time period between each support-leg exchanges. Under bounded uncertainties and bounded disturbances, the trajectory $\varphi(t)$ has deviations from the nominal orbit $\mathcal{O}$. Therefore, it is necessary to define the Euclidean distance between $\varphi(t)$ and $\mathcal{O}$ as $\operatorname{dist}(\varphi(t), \mathcal{O})$.

In order to define the conditions for gait stability under external disturbances and model uncertainties, an ultimate boundedness for the dist $(\varphi(t), \mathcal{O})$ is established. If there exist a constant $\epsilon>0$ and an open neighborhood $\mathcal{V}$ of $\mathcal{O}$ such that for every $p \in \mathcal{V}$ there exist a solution $\varphi(t)$ that satisfy $\varphi(0)=p$, and $\operatorname{dist}(\varphi(t), \mathcal{O}) \leq \epsilon, \forall t \geq 0$. The boundedness concept around the nominal orbit is illustrated in Figure 2,

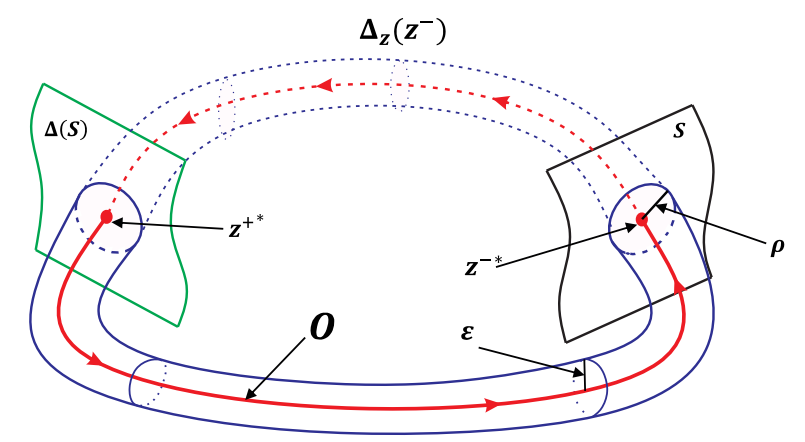

Figure 2: Neighborhood around the nominal periodic orbit

The periodicity of the gait makes the stability analysis a special case that is commonly treated with the method of Poincaré sections [17, Ch. 4]. Under undisturbed operation conditions, referred to as nominal behavior, the Poincaré method searches for periodic orbits in the evolution of the state variables. This allows to demonstrate orbital gait stability. The Poincaré method transforms the problem of finding periodic orbits into finding a fixed point on a nonlinear discrete dynamics referred as Poincaré return map. The Poincaré return map $P\left(z^{-}(k)\right)$ is built with samples of the zero dynamics state vari- 
ables just before the support-leg exchange. The undisturbed Poincaré return map is described as

$$
z^{-}(k+1)=P\left(z^{-}(k)\right),
$$

where $z^{-}(k)$ and $z^{-}(k+1)$ are the zero dynamics state variables just before the support-leg exchange at the $k$-th step and the next, respectively. The stability test of the Poincaré return map (36) is summarized in the tasks of evaluating the existence of a stable fixed point $z^{-^{*}}$, such that $z^{-*}=P\left(z^{-*}\right)$.

In order to define the stability of the Poincaré return map, a linear approximation is computed around the fixed point [34]. This is,

$$
z^{-}(k+1)=P\left(z^{-}(k)\right) \approx \Phi z^{-}(k),
$$

where $\Phi$ is the Jacobian of the Poincaré return map. The eigenvalues of $\Phi$ are used to determine the stability of $(36)$ around the fixed point $z^{-^{*}}$. Thus, if the magnitude of each eigenvalue is less than one, then the robot has an orbital periodic stable behavior with asymptotic stability. However, under uncertainties and disturbed operation condition, the asymptotic stability with convergence to a periodic orbit cannot be ensured [48]. For that reason, the distance dist $(\varphi(t), \mathcal{O})$ is utilized just before the support-leg exchange. The result is a Poincaré return map with uncertainties defined as

$$
z^{-}(k+1)=\Phi z^{-}(k)+\Xi_{\Phi}
$$

where $\Xi_{\Phi}$ represents the model uncertainties and external disturbances affecting the Poincaré return map. By definition, $\Xi_{\Phi}$ is assumed to be uniformly and absolutely bounded around the origin.

Finally, if $\psi(k)$ is the solution of (38), then the gait under model uncertainties and disturbances is stable if there exist a constant $\rho>0$ and an open neighborhood $\mathcal{U} \subset \mathcal{S}$ of the fixed point $z^{-^{*}}$ such that for every $\gamma \in \mathcal{U}$ there exist a solution $\psi(k)$ that satisfy $\psi(0)=\gamma$, and $\left\|\psi(k)-z^{-^{*}}\right\| \leq \rho, \forall k \geq 0$.

The effects of the total hybrid disturbances $\Xi_{\Phi}$ in $(38)$ are rejected through the trajectory generation strategy. This strategy is described in the next section. Since $\Xi_{\Phi}$ is bounded, the stability of the Poincaré return map (36) in the vicinity of the fixed point $z^{-*}$ is defined by the eigenvalues of $\Phi$. Thus, if the magnitude of all the eigenvalues $\lambda(\Phi)$ is less than one, the following statements are equivalent:

(i) the Poincaré return map $P\left(z^{-}(k)\right)$ is stable in a bounded neighborhood around $z^{-*}$, 
(ii) the zero dynamic state variables, $z$, converge to a bounded neighborhood around the nominal orbital behavior $\mathcal{O}$,

(iii) the full state variables, $x$, converge to a bounded neighborhood around a nominal orbital behavior, and

(iv) the gait has orbital periodic stability in a bounded neighborhood.

A trajectory generation strategy to adapt the gait to the effect of model uncertainties and external disturbances is described in the following section.

\section{Adaptive Trajectory Generation}

The trajectory generation strategy produces adaptive reference trajectories for the GPI controllers. The strategy proposed in this work is divided into three parts: first, definition of virtual holonomic constraints for a given step length; second, generation of surface virtual holonomic constraints for a given set of gait patterns; and third, a reset control law to determine the best gait pattern to fit the post-impact robot state and allows a smooth transition from the post-impact robot states to the selected gait pattern. The remaining of this section describes these three parts.

\subsection{Definition of virtual holonomic constraints for a given step length}

In order to achieve a periodic gait pattern with asymptotic stability, a nominal reference trajectory is generated using virtual holonomic constraints (VHC) [49]. VHC are imposed through feedback control with the action of actuators rather than internal forces of physical constraints. The main difference between physical and virtual holonomic constraints is that VHC can be modified on the fly without physical changes on the robot. In such a way, robustness against external disturbances and model uncertainties can be achieved through the modification of the reference trajectory defined by the nominal VHC [50].

To achieve a stable gait, the controlled joints track a set of nominal ref-

erence trajectories that are synchronized with the underactuated degree of freedom $q_{N}$. In that way, the nominal reference trajectories are designed as functions of the angle $\Theta\left(q_{s}\right)$ between the ground and the virtual link that connects the support leg-end with the hip. Since $\Theta\left(q_{s}\right)$ is a function of $q_{N}$, then synchronizing the references with $\Theta\left(q_{s}\right)$ also synchronizes the references with the underactuated degree of freedom. 
In order to obtain a smooth motion of the controlled joints, the nominal reference trajectories are determined through the Bézier polynomials

$$
\bar{q}_{d, j}\left(\Theta\left(q_{s}\right)\right)=\sum_{i=0}^{M} \beta_{i}^{j} \frac{M !}{i !(M-i) !} s^{i}(1-s)^{M-i}, \quad \forall j,
$$

where $s$ is defined in the interval $[0,1]$ and is computed with the normalization of the variable $\Theta\left(q_{s}\right)$ as

$$
s:=\frac{\Theta\left(q_{s}\right)-\Theta^{+^{*}}}{\Theta^{-*}-\Theta^{+^{*}}},
$$

$\beta_{i}^{j}$ are the coefficients of the Bézier polynomials, $M$ is the degree of the polynomials, $\Theta^{+*}$ and $\Theta^{-*}$ are the target values of the angle $\Theta\left(q_{s}\right)$ at the beginning and ending of the step, respectively. Details about the features of Bézier polynomials can be seen in [17, Ch. 6]

In order to impose a periodic behavior in the robot's hybrid dynamics, the target angles and angular velocities before and after the support-leg exchange can be linked through the reset map (8) as

$$
\left[\begin{array}{c}
q_{s}^{+*} \\
\dot{q}_{s}^{+*}
\end{array}\right]=\left[\begin{array}{c}
\Delta_{q} q_{s}^{-*} \\
\Delta_{\dot{q}}\left(q_{s}^{-*}\right) \dot{q}_{s}^{-*}
\end{array}\right]
$$

This relation reduces the amount of unknown coefficients, $\beta_{i}^{j}$, in two times the number of controlled joints, for our robot the reduction of unknown coefficients is eight.

The remaining coefficients $\beta_{i}^{j}$ in $(39)$ are numerically determined as the parameters that minimize the difference between the state variables of the robot and their target values at the end of a step. This objective is defined as

$$
J(\beta)=\left\|\left[\begin{array}{c}
q_{s}^{-*} \\
\dot{q}_{s}^{-*}
\end{array}\right]-\left[\begin{array}{c}
q_{s}^{-} \\
\dot{q}_{s}^{-}
\end{array}\right]\right\|_{2}
$$

where

$$
\beta:=\left[\begin{array}{c}
\beta_{i}^{j} \\
q_{N}^{ \pm *} \\
\dot{q}_{N}^{-*}
\end{array}\right]
$$

for $j=\{1,2,3,4\}$ and $i=\{2,3, \cdots, M\}$. 
The optimization problem can be stated as

$$
\begin{array}{cl}
\min _{\beta} & J(\beta) \\
\text { subject to } & \text { Behavioral constraints } \\
& \text { Nonlinear equality constraints } \\
& \text { Nonlinear inequality constraints } \\
& \underline{\beta} \leq \beta \leq \bar{\beta},
\end{array}
$$

where $\beta$ is the lower boundary vector and the $\bar{\beta}$ upper boundary vector. The behavioral, nonlinear equality, and nonlinear inequality constraints that produces a stable gait are described in the following sections.

\subsubsection{Behavioral constraints}

A behavioral constraint imposed by the robot's dynamics is considered in the optimization problem $(43)$. The nonlinearity and high order of the model that describes the robot's dynamics increases the complexity of the optimization problem. In order to reduce such complexity, the order of the robot's model can be enormously reduced with the assumption that a tracking control law $u$, in (18), allows tracking the VHC and zeroing the tracking error $e_{q}$. Then, the VHC induce a reduced-dimensional model, less complex than the original robot's model, defined by the zero dynamics. Thus, the zero dynamics can become the behavioral constraint of the optimization problem and, in that way, reduce the complexity of the searching process. Assuming perfect knowledge of the robot's model and without external disturbances, the zero dynamics of our robot can be extracted from (34) by replacing $\Xi=0, \forall t$. Then, the zero dynamics can be expressed as

$$
\dot{z}=f_{z}(z), \quad z^{-} \notin \mathcal{Z}
$$

where

$$
\mathcal{Z}:=\left\{\left[\begin{array}{ll}
q_{s} & \dot{q}_{s}
\end{array}\right]^{\mathrm{T}} \in \mathbb{R}^{n} \mid q_{b}=q_{d}, \dot{q}_{b}=\dot{q}_{d}\right\}
$$

\subsubsection{Nonlinear equality constraints}

Nonlinear equality constraints are imposed in the optimization problem to satisfies a target step length $s l^{*}$. Thus, the horizontal position of the swing leg-end at the beginning and ending of the step must be equal to the value of $s l^{*}$. Under the assumptions that the support leg-end is in the zero position 
and that the robot walks in the negative direction, the nonlinear equality constraints can be formulated as

$$
\begin{aligned}
p_{2}^{h^{+}}-s l^{*} & =0, \\
-p_{2}^{h^{-}}+s l^{*} & =0 .
\end{aligned}
$$

In the same way, the vertical position of the swing leg-end at the beginning and ending of the step must be equal to the ground's height $d$. Since the design is being developed for nominal conditions, the ground's height is $d=0$. Then,

$$
\begin{aligned}
& p_{2}^{v+}=0, \\
& p_{2}^{v-}=0 .
\end{aligned}
$$

\subsubsection{Nonlinear inequality constraints}

Nonlinear inequality constraints are imposed in the gait design to satisfy conditions on the reaction forces in the support leg-end. Thus, the normal reaction force $F_{1}^{v}$ is restricted to apply an unilateral constraint, such as

$$
-F_{1}^{v}<0 .
$$

On the other hand, the tangential reaction force $F_{1}^{h}$ is used to compute the ratio $F_{1}^{h} / F_{1}^{v}$, which must be less than the friction coefficient $\mu$. This is,

$$
F_{1}^{h} / F_{1}^{v}-\mu<0 .
$$

A constraint in the control signals is imposed to ensure that the torque required to track the trajectories are reachable by the actuators. In such a way, the values of the torque signals $u$ in (18) are bounded by the nominal torque of the gearmotors, $\tau_{N}$, installed in the robot. Then, the constraint is

$$
\max |u|-\tau_{N}<0 \text {. }
$$

Finally, boundary constraints are imposed to the reference trajectories to ensure that the target movements are inside of the feasible range of movement of each joint. These constraints can be described as

$$
\begin{aligned}
\max \left(\bar{q}_{d, j}\right)-q_{d, j}^{u b}<0, & \forall j, \\
-\min \left(\bar{q}_{d, j}\right)+q_{d, j}^{l b}<0, & \forall j,
\end{aligned}
$$

where $q_{d, j}^{u b}$ and $q_{d, j}^{l b}$ are the upper bound and lower bound of the $j$ th joint, respectively. The following section presents a recurrent method to generate a set of gait patterns with different step length. 


\subsection{Generation of a surface of virtual holonomic constraints for a set of gait patterns}

The VHC approach developed in Sec. 5.1 is used to generate a set of gait patterns that allows to reconfigure the VHC through software manipulation [12, 33. In the method presented by [17], VHC are functions of the evolution angle $\Theta\left(q_{s}\right)$. Typically, VHC are updated only after each support-leg exchange, right before the swing phase. This feature implies a limitation in the classical VHC approach, because reaction to disturbances are taken just at the support leg exchange.

In this work, we propose to dynamically adjust VHC during the swing phase by adding the step length $s l$ as an additional control variable. To this end, a set of gait patterns is defined for $0.13 \mathrm{~m} \leq s l \leq 0.20 \mathrm{~m}$. After all gait patterns have been defined, the results of each combination of $s l$ and $\Theta\left(q_{s}\right)$ are used to fit four smooth $5 \times 5$ polynomials that allow to continuously modify the step length. The result of the fitting is shown in the Figure 3 . These surfaces are defined by $q_{d_{i}}^{a u x}\left(\Theta\left(q_{s}\right), s l\right)$, which are the reference trajectories for each controlled joint.

Including the step length as a control variable to define the reference trajectories gives the trajectory generator the flexibility to reject model uncertainties and external disturbances in both continuous and discrete of a bipedal robot's dynamics. In such a way, the following section develops a reset control law to deal with disturbances that apart the robot from its nominal walking conditions.

\subsection{Reset control law to reject disturbances at the support-leg exchange}

A reset control law is proposed to reject disturbances at the support-leg exchange. This reset control law combines a reset trajectory with a smooth transition from the post-impact robot states to the selected gait pattern. The reset trajectory uses an optimization process to find the gait pattern that best fit the configuration of the robot after each support-leg exchange. In a complementary fashion, a smooth functions uses the passive trend of the post-impact states to perform a transition from the state of the robot after the support-leg exchange to the target trajectory. These two parts of the reset control law are described in the remainder of this section. 

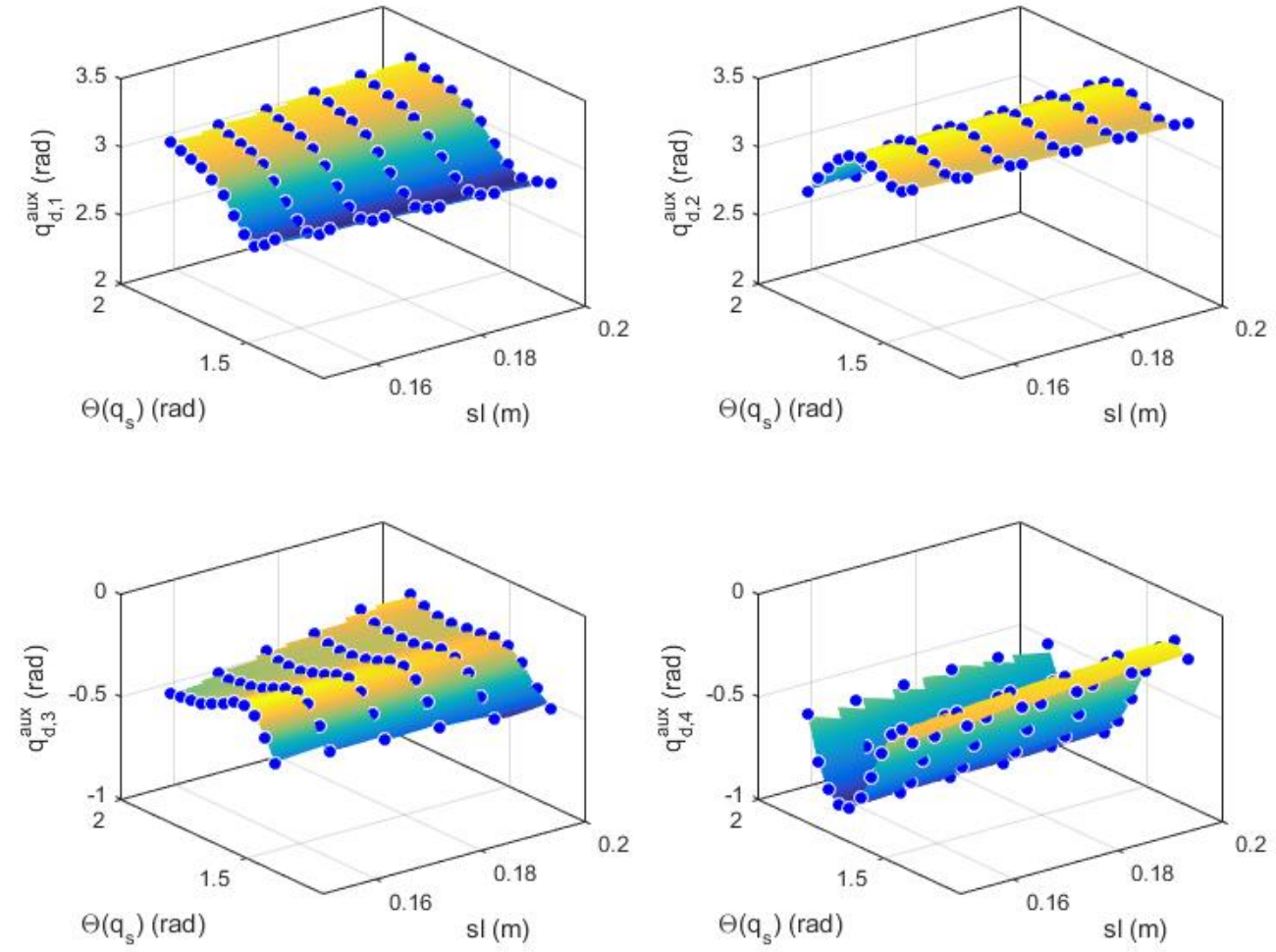

Figure 3: Trajectory reference surfaces based on $\Theta\left(q_{s}\right)$ and step length, sl.

\subsubsection{Reset trajectory optimization at the transition of the support-leg ex- change}

The main limitation associated with the traditional use of VHC is the need of continuity on the trajectory references to ensure zero tracking error after the support-leg exchange. This limitation implies that, after the support leg exchange, the robot should be in a geometric configuration that coincides with the reference trajectory of the coming swing phase. This condition is almost never satisfied in uncertain walking conditions; therefore, the tracking errors at the beginning of swing phase are not zero. Despite the fact that the tracking controller can rapidly reduce the tracking error, a transitory response affects the controlled joints suffering sudden changes. These sudden 
changes can produce a violation of the contact constraints on the support leg-end and cause the robot to take off the ground or slide.

In order to address the continuity limitations of the VHC approach in the support leg-exchange, this work presents an optimization strategy that minimizes the tracking errors after the support leg exchange and, therefore, minimizes the effects of the transitory responses. To this end, let us find a post-impact step length $s l_{d}^{+}$that minimizes the tracking error right after the support-leg exchange. The post-impact tracking error is defined as the difference between a set of references $q_{d_{i}}^{a u x}\left(\Theta\left(q_{s}\right)^{+}, s l_{d}^{+}\right)$and the robot's configuration $q_{b_{i}}^{+}$. The optimization problem is formulated as

$$
\begin{array}{ll}
\min _{s l_{d}^{+}} & \sum_{i=1}^{4}\left\|q_{d_{i}}^{\text {aux }}\left(\Theta\left(q_{s}\right)^{+}, s l_{d}^{+}\right)-q_{b_{i}}^{+}\right\|_{2}^{2} \\
\text { s.t. } & 0.13 \leq s l_{d}^{+} \leq 0.20 .
\end{array}
$$

The optimal post-impact step length $\left(s l_{d}^{+}\right)^{*}$ is found by solving the optimization problem (55) online after each support-leg exchange. This solution is used as the initial step length in each swing phase. Then, a dynamic transition between the initial step length $\left(s l_{d}^{+}\right)^{*}$ and the target step length $s l_{d}$ is determined by the transfer function

$$
s l_{r}=\frac{1}{\tau_{s l} s+1} s l_{d}
$$

with the initial condition $\left(s l_{d}^{+}\right)^{*}$. The transfer function (56) incorporates a step length dynamics to the trajectory definition. This dynamics is defined to be stable and with a steady state gain equals to one. The corresponding time constant $\tau_{s l}$ is appropriately chosen to guarantee that the step length $s l_{r}$ converges to its stationary value before the next support-leg exchange. This work uses the condition $5 \tau_{s l} \leq t_{\text {avg }} / 2$, where $t_{\text {avg }}$ is the average time that a swing phase takes. The reference polynomials evaluated at $s l_{r}$ in (56) produce an auxiliary set of trajectories $q_{d_{i}}^{a u x}\left(\Theta\left(q_{s}\right), s l_{r}\right)$. Such trajectories are illustrated in Figure 4. This figure also shows the transition of the reference from $\left(s l_{d}^{+}\right)^{*}$ to the target step length $s l_{d}$. With the proposed approach, the transitory responses are minimized and the best gait pattern is defined for the robot's state after each support-leg exchange. The next section presents a strategy to connect the post-impact robot configuration with the target trajectory. 


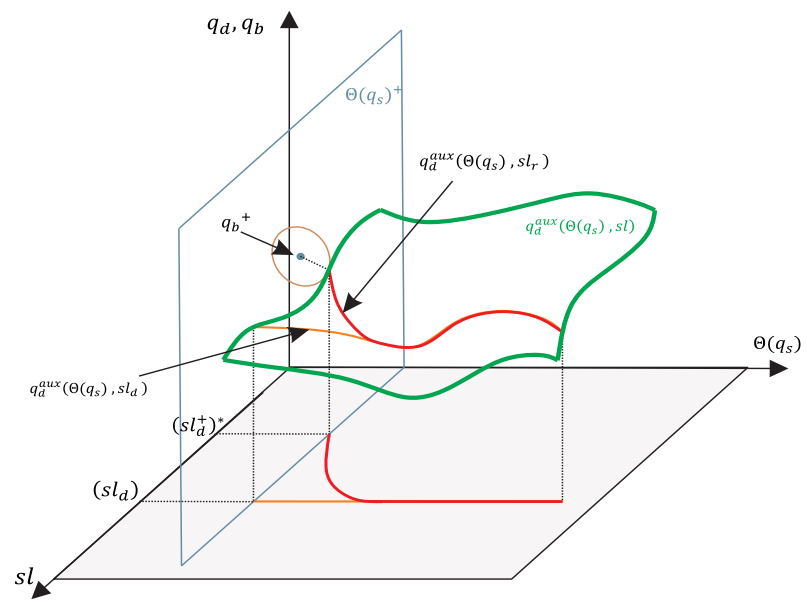

Figure 4: Optimal reset reference trajectory.

\subsubsection{Smooth transition from the passive dynamics to the target trajectory}

In order to provide continuity to the robot's motion on uncertain walking conditions, this work proposes a smooth transition function that drives the robot's states from the post-impact state to the target reference trajectories. This smooth transition benefits estimation of the state variables because jumps in the controlled joints are avoided and the state variables estimation remains in an invariant set [51, p. 68].

Under uncertain discrete dynamics, it is not possible to ensure that the robot's states before the support-leg exchange are in their nominal values, defined here as

$$
\bar{x}^{-}=\left[\begin{array}{c}
q_{s}^{-*} \\
\dot{q}_{s}^{-*}
\end{array}\right] .
$$

In that way, let us define the real pre-impact states as

$$
x^{-}=\bar{x}^{-}+\varepsilon^{-},
$$

where $\varepsilon^{-}$is the offset between the nominal pre-impact states and the real ones. Then, the post-impact states are defined by an extension of the reset map in (7) as

$$
\begin{aligned}
x^{+} & =\Delta\left(x^{-}\right)+\gamma_{\Delta}, \\
\left(\bar{x}^{+}+\varepsilon^{+}\right) & =\Delta\left(\bar{x}^{-}+\varepsilon^{-}\right) .
\end{aligned}
$$


The above result shows that even though the reference trajectories are designed as in (43) to satisfy that $J(\beta) \rightarrow 0$, it is unsure whether these conditions will be satisfied on uncertain terrain.

In order to avoid the sudden changes that could be produced by the uncertain terrain, the post-impact angles $q_{b}^{+}$and the angular velocities $\dot{q}_{b}^{+}$are used to perform a smooth transition from the post-impact states to the nominal reference trajectories. To this end, let us define a passive trend function that will act as a passive reference just after the support-leg exchange. This is,

$$
\vartheta_{j}=\left(\dot{q}_{b}^{+}\right)_{j} \tau+\left(q_{b}^{+}\right)_{j}, \quad \forall j
$$

considering the reset map in (8), (61) takes the form

$$
\vartheta_{j}=\left(\Delta_{\dot{q}}\left(q_{s}^{-}\right) \dot{q}_{s}^{-}\right)_{j} \tau+\left(\Delta_{q} q_{s}^{-}\right)_{j}, \quad \forall j,
$$

where $\tau$ is a time variable that is reset to zero after each support-leg exchange. The functions $\vartheta_{j}$ in $(62)$ are used as the main passive reference just after the support-leg exchange. Then, a transition from $\vartheta$ to the trajectory $q_{d}^{a u x}\left(\Theta\left(q_{s}\right), s l_{r}\right)$ is performed with the use of the function

$$
q_{d, j}=q_{d, j}^{a u x}+\left(\vartheta_{j}-q_{d, j}^{a u x}\right) B_{z}\left(t_{a u x}\right), \quad \forall j,
$$

where $q_{d, j}^{a u x}$ is the short of $q_{d, j}^{a u x}\left(\Theta\left(q_{s}, s l_{r}\right)\right), B_{z}\left(t_{a u x}\right)$ is a smooth function that changes from one to zero in a given period of time. The function $B_{z}\left(t_{\text {aux }}\right)$ is defined by a third-order Bézier polynomial as

$$
B_{z}\left(t_{\text {aux }}\right)=\sum_{i=0}^{3} b_{i} \frac{3 !}{i !(3-i) !} t_{\text {aux }}^{i}\left(1-t_{\text {aux }}\right)^{3-i}, \quad 0 \leq t_{\text {aux }} \leq 1,
$$

where $b_{i}$ are the Bézier coefficients, $t_{\text {aux }}$ is the auxiliary time variable, which is defined in such a way that (64) converges to zero in a time significantly lower than the duration of the nominal swing phase. In other words,

$$
t_{\text {aux }}=k_{\tau} \tau
$$

where $k_{\tau}$ is a constant that sets the transition period of the reference to $1 / k_{\tau}$. The Bézier polynomial satisfy the boundary conditions

$$
B_{z}(0)=b_{0}=1, \quad B_{z}(1)=b_{3}=0
$$




$$
\left.\left(\frac{\partial B_{z}\left(t_{\text {aux }}\right)}{\partial t_{\text {aux }}}\right)\right|_{t_{\text {aux }}=0}=3\left(b_{1}-b_{0}\right)=0
$$

and

$$
\left.\left(\frac{\partial B_{z}\left(t_{\text {aux }}\right)}{\partial t_{\text {aux }}}\right)\right|_{t_{\text {aux }=1}}=3\left(b_{3}-b_{2}\right)=0 .
$$

Figure 5 shows the evolution of a reference $q_{d, j}$ from the trend function $\vartheta_{j}$ to the target trajectory $q_{d, j}^{a u x}$. As observed, the proposed smooth transition technique produces zero tracking error and zero first time-derivative error at the beginning of each step.

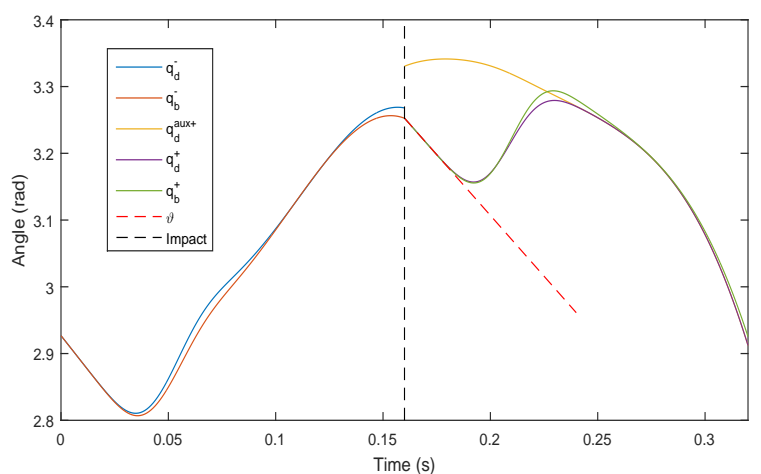

Figure 5: Transference from the trend to the designed trajectory.

\section{Simulation and Experimental Test}

Numerical simulations are performed to evaluate the robustness of the proposed compound control strategy. Table 1 shows the physical parameters of the robot. The controller tuning is based on a third-degree time polynomial approximation of the lumped disturbances $\gamma_{\xi}\left(q_{s}, \dot{q}\right)$ in (16). Following the protocol proposed in [52], the coefficients $k_{i, j}$ of the GPI controller are tuned to provide a stable and dominant behavior in the characteristic polynomial (33). The corresponding closed-loop roots are found to be $-40,-50,-60$, $-65,-70$, and -75 . The time constant $\tau$ in the transfer function (56) is set at $\tau_{s l}=0.05$.

The gait of the robot is tested through the simulation with four operation conditions. The first operation condition is a horizontal walk on flat terrain. The second operation condition corresponds to a walk on a terrain 
Table 1: Physical parameters of the robot (Fig. 1).

\begin{tabular}{|c|c|c|c|}
\hline Model Parameter & Unit & Link & Value \\
\hline \multirow{3}{*}{ Mass } & \multirow{3}{*}{$\mathrm{kg}$} & Torso & 2.5 \\
\cline { 3 - 4 } & & Tight & 1.0 \\
\cline { 3 - 4 } & \multirow{3}{*}{ Length } & Shin & 0.4 \\
\hline \multirow{3}{*}{ Inertia } & $\mathrm{m}$ & Torso & 0.291 \\
\cline { 3 - 4 } & & Tight & 0.185 \\
\cline { 3 - 4 } & \multirow{3}{*}{$\mathrm{kg} \cdot \mathrm{m}^{2}$} & Shin & 0.185 \\
\hline \multirow{2}{*}{$\begin{array}{c}\text { Mass } \\
\text { center* }\end{array}$} & & Torso & 0.014 \\
\cline { 3 - 4 } & & Shight & 0.010 \\
\hline \multirow{2}{*}{$\mathrm{m}$} & Torso & 0.002 \\
\cline { 3 - 4 } & & Tight & 0.062 \\
\cline { 3 - 4 } & & Shin & 0.055 \\
\hline
\end{tabular}

*Mass center with respect to the proximal joints.

with height changes in uphill and downhill directions. The third operation condition is a horizontal walk on flat terrain with external force disturbances. Robustness against external disturbance is evaluated in this operation condition. The fourth operation condition corresponds to a horizontal walk on flat terrain with variations of the robot parameters. Robustness against model mismatching is evaluated in this operation condition. Finally, a physical experimentation on the prototype of a planar biped robot is developed with walking over a flat terrain.

\subsection{Simulation under nominal conditions}

The first simulation evaluates the compound controller under nominal conditions, namely, gait on flat terrain without external disturbances and without model uncertainties. Figure 6 shows the trajectory tracking and the control torques required to drive the robot to stable walking. As observed, the controlled joints, $q_{s, 1}, \ldots, q_{s, 4}$, effectively track the trajectory references, $q_{d, 1}, \ldots, q_{d, 4}$, with the maximum values of the torques in an admissible range of $\pm 5 \mathrm{~N} \cdot \mathrm{m}$. Figure 7 shows the reaction forces on the support leg-end. The positive sign of the normal force $F_{1}^{v}$ proves that the robot does not take off from the ground. In addition, the ratio between the tangential and the normal reaction forces is less than the friction coefficient $\mu=0.6$, which guarantees that the support leg does not slip. These two characteristics of 
the reaction forces demonstrate that the unilateral constraints are satisfied.
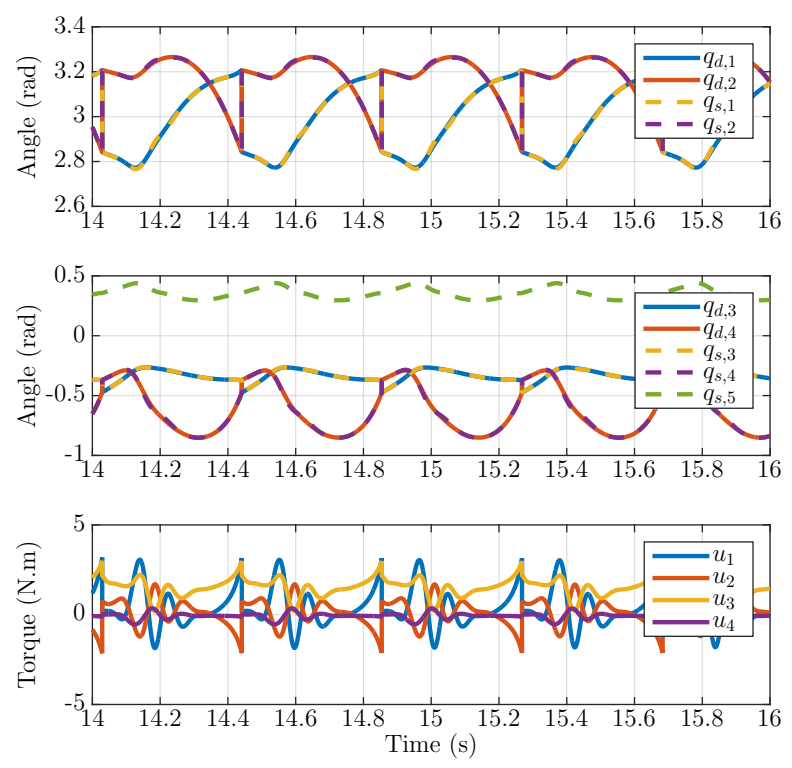

Figure 6: Tracking references and control signals.

The Poincaré return map is computed with the hybrid zero dynamics state variables and the robot walking on nominal conditions. For this robot, the Jacobian $\Phi$ in $(38)$ is

$$
\Phi=\left[\begin{array}{cc}
-0.2719 & -0.0065 \\
-0.5637 & 0.7718
\end{array}\right],
$$

and the corresponding magnitudes of the eigenvalues are

$$
|\lambda(\Phi)|=\left[\begin{array}{l}
0.2754 \\
0.7753
\end{array}\right] .
$$

Given that the magnitudes of those eigenvalues are less than one, the total walking is asymptotically stable with periodic behavior.

\subsection{Simulation on uneven terrain}

The second simulation is performed over an uneven terrain with slopes of $+3^{\circ}$ (uphill), $0^{\circ}$ (flat), and $-3^{\circ}$ (downhill). Considering that the gait patterns 

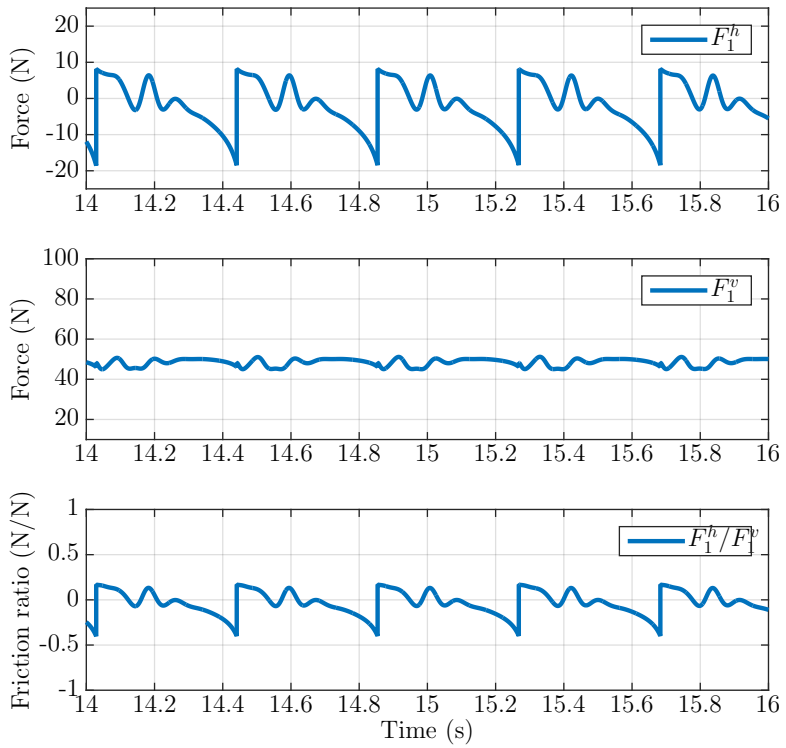

Figure 7: Reaction forces.

were designed for a flat terrain, the evaluation over an uneven ground entails a challenging robustness test that reveals the capabilities of the robot to walk over uncertain terrain. Figure 8 a) shows a stick diagram of the robot walking over flat, uphill, and downhill terrains. The robot maintains balance and satisfies all physical constraints.

In order to estimate the robustness of the proposed compound controller against uneven terrain, simulations of the robot facing discrete changes in the terrain's height are performed. The resulting maximum terrain's height variation is found to be within the range $[-10,10] \mathrm{mm}$. Figure $8 \mathrm{~b}$ ) shows the stick diagram of the gait evolution over a terrain with discrete height changes. The results of the simulations over uneven terrain show the capabilities of the proposed control to increase the robustness of the walking over uneven terrain.

\subsection{Simulation with external disturbances}

The third group of simulations demonstrate the robustness of the compound controller against external disturbances. The robot model is exposed 

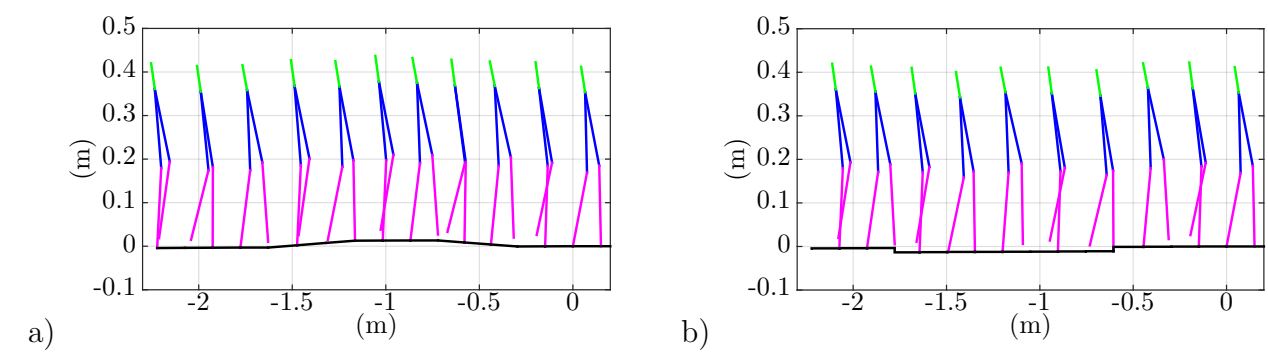

Figure 8: Stick diagram with the evolution of the walking over uneven terrain.a) uphill and downhill b) stairs down and stairs up.

to persistent and impulsive disturbances. In order to test the robot's behavior with persistent disturbances, constant external forces are applied on the robot's hip as in [53]. A positive persistent disturbance of [0.4 0$] \mathrm{N}$ acts in the walking direction and produces an absolute forward velocity of $0.53 \mathrm{~m} / \mathrm{s}$, which is higher than the absolute nominal velocity of $0.42 \mathrm{~m} / \mathrm{s}$. An equivalent persistent disturbance is applied on the opposite direction. Under this external force, the walking converges to a lower speed of $0.20 \mathrm{~m} / \mathrm{s}$. Figure 9 shows the average horizontal velocity of the robot's hip during the walk. Additional simulations are performed with the robot walking on nominal conditions for ten steps. Then, disturbance torques are applied for $0.02 \mathrm{~s}$ in the middle of the swing phase with a magnitude of $\pm 40 \%$ of the maximum torque available on the motors, which is $5 \mathrm{~N} \cdot \mathrm{m}$. Figure 9 also shows how the robot recovers its nominal average velocity after the external disturbances demonstrating the effectiveness of the disturbance rejection strategy. Figure 10 shows the phase portrait with the evolution of the states of the robot after the external disturbance. In this figure, the phase portraits show a transient response followed by a convergence to a limit cycle. This demonstrates the effective rejection of the external disturbance by the proposed controller.

\subsection{Simulation with parameter uncertainties}

The final simulation evaluates the robustness against parameter uncertainties (internal disturbances) with variation of $\pm 20 \%$ on the mass of the torso, tights, and shins. Figure 11 shows the evolution of $\Theta\left(q_{s}\right)$ and $\dot{\Theta}\left(q_{s}\right)$ for the robot walking with time-invariant masses of $80 \%, 100 \%$, and $120 \%$ of their nominal values (Table 1). All the simulations start at the same initial conditions and all them have the same step length target. This figure 


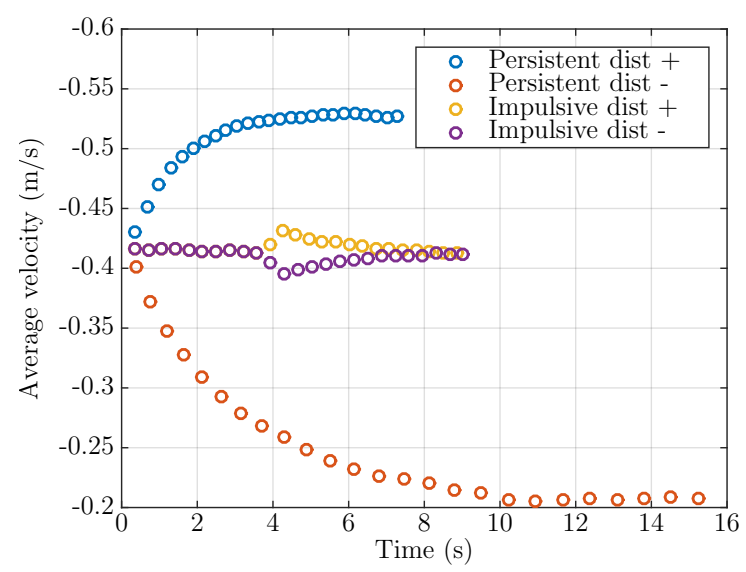

Figure 9: Hip's horizontal average velocity.

shows the expected adaptive behavior on the step length as a response to the disturbances produced by the parameter uncertainties.

\subsection{Physical experiment}

In order to verify the effectiveness of the proposed GPI controller and the trajectory generation strategy, a physical experiment is performed on a planar biped robot prototype - refereed to as Saurian. The complete scheme of control is presented in the Figure 12. The physical prototype is exposed to the persistent external disturbances of the central bar and model uncertainties, which include but are not limited to backlash, joint compliance, Coulomb friction, and parameter uncertainties.

The results shown in Figure 13 demonstrate that the four controlled joints $\left(h_{l}, h_{r}, k_{l}, k_{r}\right)$ closely track the trajectory references generated for a fixed step length of $15 \mathrm{~cm}$ as described in Sec. 5. The periodic behavior of the underactuated degrees of freedom $\left(q_{T}\right)$ along with the periodic behavior of the controlled joints demonstrate that the proposed control strategy produces a stable walking.

A sequence of photos taken from a video of the Saurian walking with the proposed control law are shown in Figure 14. The effective experiments performed on Saurian confirm that the proposed compound control strategy produces robust stable gaits even walking under model uncertainties and external disturbances. 

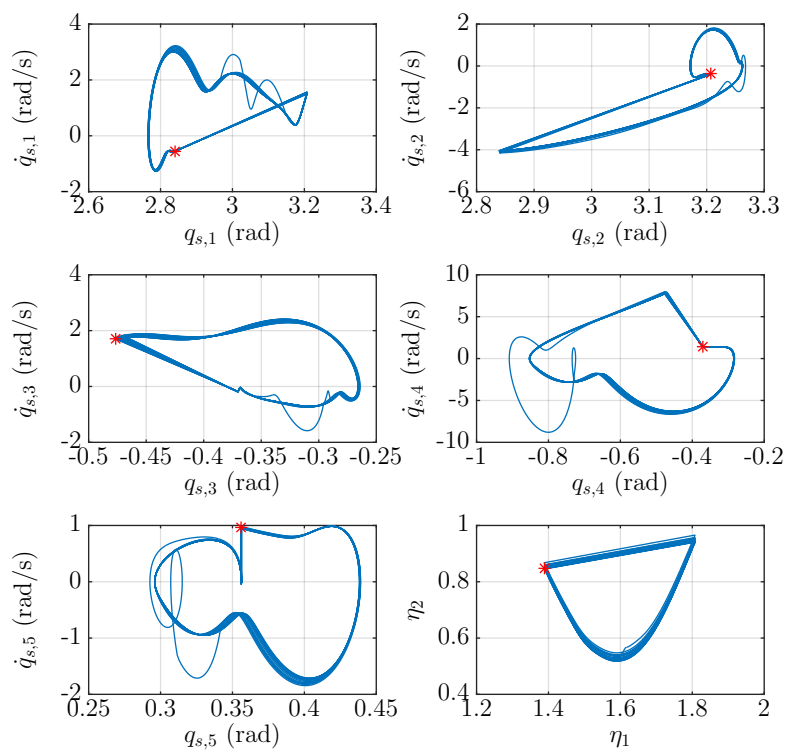

Figure 10: States convergence to nominal cycles after external disturbance. The initial conditions are marked with red stars

In order to estimate the energy cost of the robot's gait, a metric based on the specific mechanical cost of transport is utilized. For a nominal step, the specific mechanical cost of transport is defined as the ratio between the energy consumed in a step and the product of the step length and the robot's weight [54]. This can be expressed as

$$
C_{T}=\frac{1}{m g\left|\operatorname{hip}^{h}\left(t_{f}\right)-\operatorname{hip}^{h}\left(t_{0}\right)\right|} \int_{t_{0}}^{t_{f}} P_{w} d t,
$$

where

$$
P_{w}=\left\{\begin{array}{lll}
\dot{q}_{s}^{\mathrm{T}} B u, & \text { if } \quad \dot{q}_{s}^{\mathrm{T}} B u>0, \\
0, & \text { if } \quad & \dot{q}_{s}^{\mathrm{T}} B u \leq 0,
\end{array}\right.
$$

$\operatorname{hip}^{h}(t)$ is the horizontal position of the hip at time $t, m$ is the total mass of the robot, $g$ is the gravity constant, $t_{0}$ and $t_{f}$ are the time at the beginning and end of a step, respectively.

The specific mechanical cost of transport is presented in Table 2, When compared to other testbeds with equivalent degrees of mobility, the proposed 


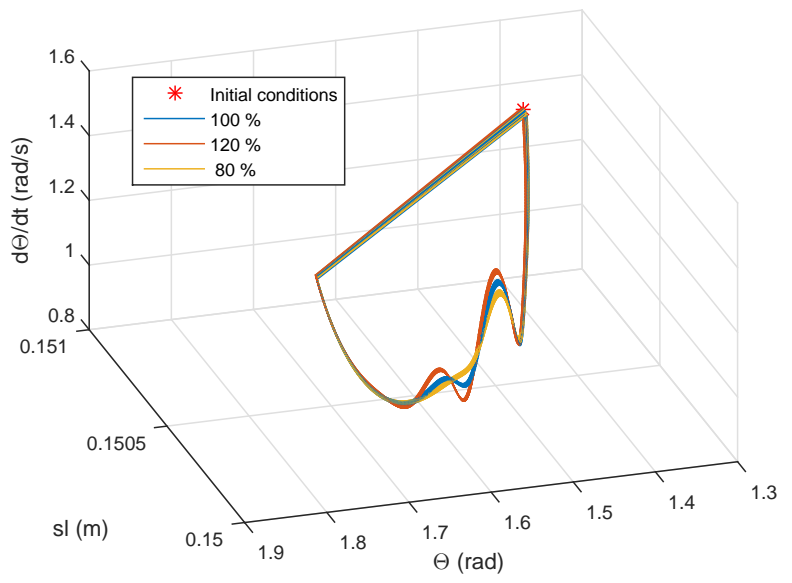

Figure 11: Parameters uncertainties.

strategy has a relatively low $C_{T}$ demonstrating an efficient energy use.

Table 2: Specific mechanical cost of transport $C_{T}$.

\begin{tabular}{|l|c|}
\hline Testbed & $C_{T}$ \\
\hline RABBIT [55] & 0.380 \\
\hline MABEL [56] & 0.290 \\
\hline Saurian (Robust Compound GPI controller) & $\mathbf{0 . 1 2 9}$ \\
\hline Cornell Biped [54] & 0.055 \\
\hline
\end{tabular}

\section{Conclusion}

A robust compound control strategy for dynamic bipedal robots was developed in this work. The compound control strategy consists of a nonswitching robust GPI controller (inner loop) and an adaptive trajectory generation strategy (outer loop). The adaptive trajectory generator was developed to provide robustness against external disturbances and model uncertainties. This adaptive trajectory generator minimizes the disturbance and uncertainty effects by updating the step length of the gait patter. The robust GPI controller effectively tracks reference trajectories and rejects lumped disturbance signals that act on the actuated joints. The results demonstrate 


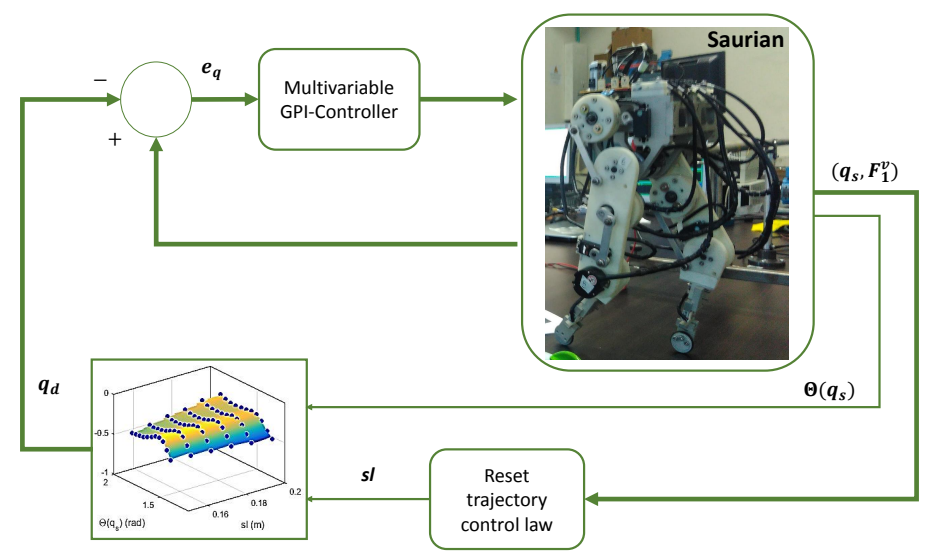

Figure 12: Complete robust compound control scheme

that the proposed compound controller achieved periodic and asymptotic stable walking on nominal operation conditions. Additionally, under disturbed operation conditions the numerical and physical evaluations show stable behavior on a neighborhood around the nominal gait.

This work contributes to two specific areas within dynamical bipedal robotics: trajectory tracking control and trajectory generation. In terms of trajectory tracking control, the use of a simplified model allows the dynamic decoupling of multivariate disturbances. The implementation of a robust GPI controller for a hybrid nonlinear, multi-input multi-output and underactuated system, provides an effective trajectory tracking and the rejection of external disturbances and model uncertainties without the explicit use of state observers. In terms of trajectory generation, a dynamic adjustment of VHC during the swing phase as integral part of the adaptive trajectory generation strategy is developed. This is achieved by adding the step length as a control variable. The result is the rejection of model uncertainties and external disturbances such as terrain variations. The adaptive trajectory generator minimize the effect of transitory responses at the support-leg exchange and drives the robot from its passive dynamics to a target gait pattern.

Ongoing research endeavors include feedback control of the forward average velocity and the design of gait patters for uphill and downhill terrains. 

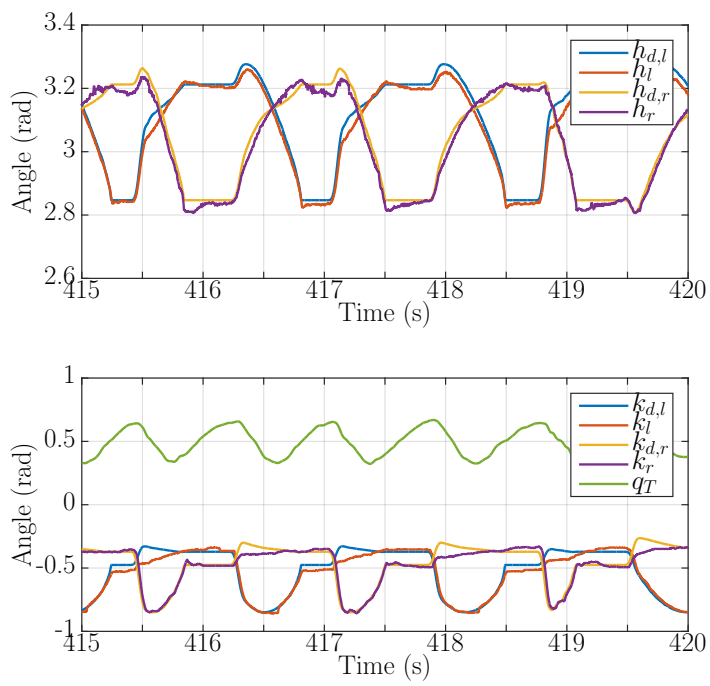

Figure 13: Experimental results of GPI tracking control.

\section{Acknowledgment}

This research was supported by the Office of Research at the National University of Colombia, Bogota campus, projects 28340 and 37662, and the National Science Foundation NRT-IGE grant 1633426. The first author was supported by the Fulbright Commission in Colombia and Colombian Administrative Department of Science, Technology and Innovation-Colciencias.

[1] T. McGeer, Passive walking with knees, in: Robotics and Automation, 1990. Proceedings., 1990 IEEE International Conference on, IEEE, 1990, pp. 1640-1645.

[2] G. Nelson, A. Saunders, N. Neville, B. Swilling, J. Bondaryk, D. Billings, C. Lee, R. Playter, M. Raibert, Petman: A humanoid robot for testing chemical protective clothing, Journal of the Robotics Society of Japan 30 (4) (2012) 372-377.

[3] M. Johnson, B. Shrewsbury, S. Bertrand, D. Calvert, T. Wu, D. Duran, D. Stephen, N. Mertins, J. Carff, W. Rifenburgh, et al., Team ihmc's lessons learned from the darpa robotics challenge: finding data in the rubble, Journal of Field Robotics 34 (2) (2017) 241-261. 


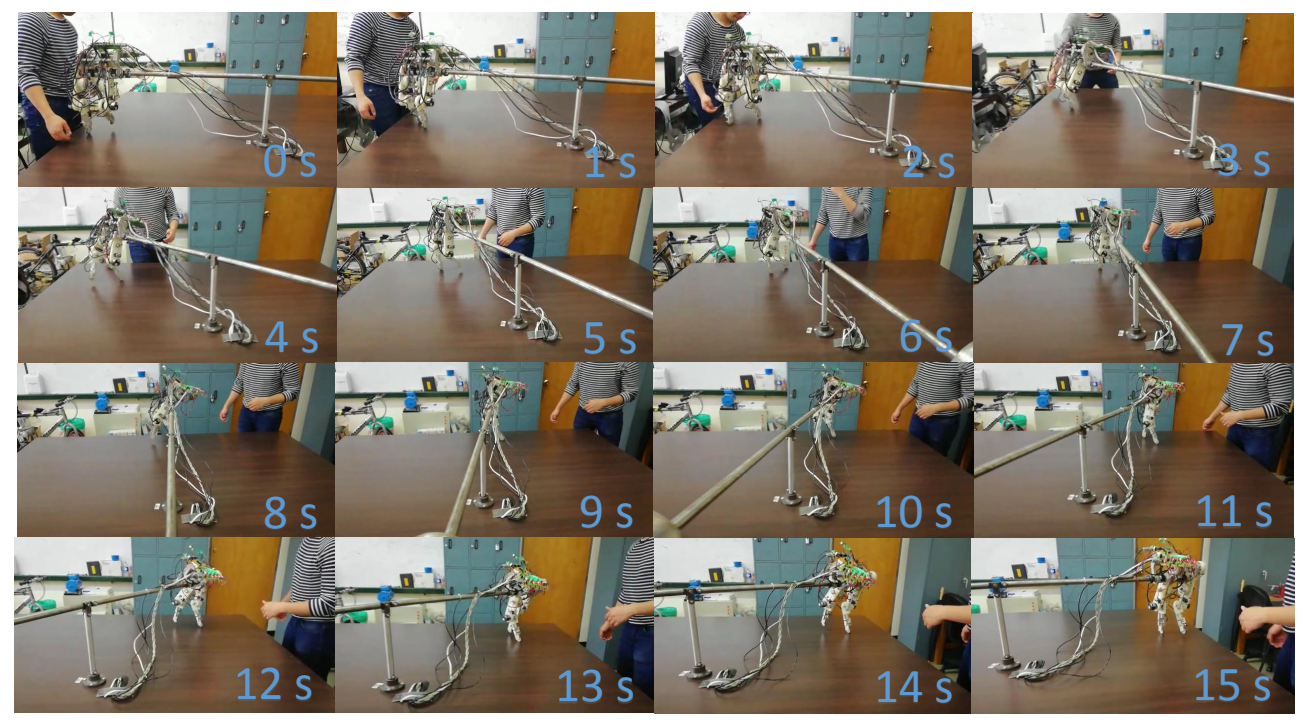

Figure 14: Snapshots sampled with a period of $1.0 \mathrm{~s}$. See video in https://youtu.be/ dmWx3JFrf7c

[4] N. A. Radford, P. Strawser, K. Hambuchen, J. S. Mehling, W. K. Verdeyen, A. S. Donnan, J. Holley, J. Sanchez, V. Nguyen, L. Bridgwater, et al., Valkyrie: Nasa's first bipedal humanoid robot, Journal of Field Robotics 32 (3) (2015) 397-419.

[5] T. Gurriet, S. Finet, G. Boeris, A. Hereid, O. Harib, M. Masselin, J. W. Grizzle, A. D. Ames, Towards restoring locomotion for paraplegics: Realizing dynamically stable walking on exoskeletons, in: Robotics and Automation (ICRA), IEEE International Conference on, IEEE, 2018.

[6] H. Zhao, J. Horn, J. Reher, V. Paredes, A. D. Ames, First steps toward translating robotic walking to prostheses: a nonlinear optimization based control approach, Autonomous Robots 41 (3) (2017) 725-742.

[7] Y. Sakagami, R. Watanabe, C. Aoyama, S. Matsunaga, N. Higaki, K. Fujimura, The intelligent asimo: System overview and integration, in: Intelligent Robots and Systems, 2002. IEEE/RSJ International Conference on, Vol. 3, IEEE, 2002, pp. 2478-2483.

[8] M. Vukobratović, B. Borovac, Zero-moment point-thirty five years of its life, International Journal of Humanoid Robotics 1 (01) (2004) 157-173. 
[9] S. Kajita, H. Hirukawa, K. Harada, K. Yokoi, Introduction to humanoid robotics, Vol. 101, Springer, 2014.

[10] W.-L. Ma, S. Kolathaya, E. R. Ambrose, C. M. Hubicki, A. D. Ames, Bipedal robotic running with durus-2d: Bridging the gap between theory and experiment, in: Proceedings of the 20th International Conference on Hybrid Systems: Computation and Control, ACM, 2017, pp. 265-274.

[11] C. Hubicki, J. Grimes, M. Jones, D. Renjewski, A. Spröwitz, A. Abate, J. Hurst, Atrias: Design and validation of a tether-free 3d-capable spring-mass bipedal robot, The International Journal of Robotics Research 35 (12) (2016) 1497-1521.

[12] C. Canudas-de Wit, On the concept of virtual constraints as a tool for walking robot control and balancing, Annual Reviews in Control 28 (2) (2004) 157-166.

[13] C. Chevallereau, G. Abba, Y. Aoustin, F. Plestan, E. Westervelt, C. C. de Wit, J. Grizzle, Rabbit: A testbed for advanced control theory, IEEE Control Systems Magazine 23 (5) (2003) 57-79.

[14] K. Sreenath, H.-W. Park, I. Poulakakis, J. W. Grizzle, A compliant hybrid zero dynamics controller for stable, efficient and fast bipedal walking on mabel, The International Journal of Robotics Research 30 (9) (2011) 1170-1193.

[15] K. A. Hamed, J. W. Grizzle, Reduced-order framework for exponential stabilization of periodic orbits on parameterized hybrid zero dynamics manifolds: Application to bipedal locomotion, Nonlinear Analysis: Hybrid Systems 25 (2017) 227-245.

[16] E. R. Westervelt, J. W. Grizzle, D. E. Koditschek, Hybrid zero dynamics of planar biped walkers, Automatic Control, IEEE Transactions on 48 (1) (2003) 42-56.

[17] E. R. Westervelt, J. W. Grizzle, C. Chevallereau, J. H. Choi, B. Morris, Feedback control of dynamic bipedal robot locomotion, Vol. 28, CRC press, 2007. 
[18] H. Dai, R. Tedrake, L 2-gain optimization for robust bipedal walking on unknown terrain, in: Robotics and Automation (ICRA), 2013 IEEE International Conference on, IEEE, 2013, pp. 3116-3123.

[19] B. Griffin, J. Grizzle, Walking gait optimization for accommodation of unknown terrain height variations, in: American Control Conference (ACC), 2015, IEEE, 2015, pp. 4810-4817.

[20] B. Griffin, J. Grizzle, Nonholonomic virtual constraints and gait optimization for robust walking control, The International Journal of Robotics Research 36 (8) (2017) 895-922.

[21] A. D. Ames, K. Galloway, K. Sreenath, J. W. Grizzle, Rapidly exponentially stabilizing control lyapunov functions and hybrid zero dynamics, IEEE Transactions on Automatic Control 59 (4) (2014) 876-891.

[22] S.-C. Hsu, X. Xu, A. D. Ames, Control barrier function based quadratic programs with application to bipedal robotic walking, in: American Control Conference (ACC), 2015, IEEE, 2015, pp. 4542-4548.

[23] Q. Nguyen, K. Sreenath, Optimal robust control for bipedal robots through control lyapunov function based quadratic programs., in: Robotics: Science and Systems, 2015.

[24] S. Kolathaya, A. D. Ames, Parameter to state stability of control lyapunov functions for hybrid system models of robots, Nonlinear Analysis: Hybrid Systems 25 (2017) 174-191.

[25] S. Veer, Rakesh, I. Poulakakis, Input-to-state stability of periodic orbits of systems with impulse effects via poincaré analysis.

[26] J. C. Horn, A. Mohammadi, K. A. Hamed, R. D. Gregg, Hybrid zero dynamics of bipedal robots under nonholonomic virtual constraints, IEEE Control Systems Letters 3 (2) (2019) 386-391.

[27] K. Galloway, K. Sreenath, A. D. Ames, J. W. Grizzle, Torque saturation in bipedal robotic walking through control lyapunov function-based quadratic programs, IEEE Access 3 (2015) 323-332.

[28] A. Mohammadi, S. Fakoorian, J. C. Horn, D. Simon, R. D. Gregg, Hybrid nonlinear disturbance observer design for underactuated bipedal 
robots, in: 2018 IEEE Conference on Decision and Control (CDC), IEEE, 2018, pp. 1217-1224.

[29] J. Arcos-Legarda, J. Cortes-Romero, A. Beltran-Pulido, A. Tovar, Hybrid disturbance rejection control of dynamic bipedal robots, Multibody System Dynamics (2019) 1-26.

[30] T. L. Brown, J. P. Schmiedeler, Gait transitions and disturbance response for planar bipeds with reaction wheel actuation, in: Intelligent Robots and Systems (IROS), 2016 IEEE/RSJ International Conference on, IEEE, 2016, pp. 3393-3398.

[31] T. L. Brown, J. P. Schmiedeler, Reaction wheel actuation for improving planar biped walking efficiency, IEEE Transactions on Robotics 32 (5) (2016) 1290-1297.

[32] B. G. Buss, K. A. Hamed, B. A. Griffin, J. W. Grizzle, Experimental results for $3 \mathrm{~d}$ bipedal robot walking based on systematic optimization of virtual constraints, in: American control conference, 2016.

[33] C. Chevallereau, J. W. Grizzle, C.-L. Shih, Asymptotically stable walking of a five-link underactuated 3-d bipedal robot, Robotics, IEEE Transactions on 25 (1) (2009) 37-50.

[34] J. Arcos-Legarda, J. Cortes-Romero, A. Tovar, Generalized proportional integral control for aperiodic gait stabilization of a bipedal robot with seven degrees of freedom, in: Latin American Conference of Automatic Control, 2016.

[35] Z. Gao, Active disturbance rejection control: a paradigm shift in feedback control system design, in: American Control Conference, 2006, IEEE, 2006, pp. 7-pp.

[36] J. Arcos-Legarda, J. Cortes-Romero, A. Tovar, Active disturbance rejection control based on generalized proportional integral observer to control a bipedal robot with five degrees of freedom, in: American Control Conference (ACC), 2016, IEEE, 2016.

[37] K. Guo, J. Wei, J. Fang, R. Feng, X. Wang, Position tracking control of electro-hydraulic single-rod actuator based on an extended disturbance observer, Mechatronics 27 (2015) 47-56. 
[38] X. Shi, S. Chang, Extended state observer-based time-optimal control for fast and precise point-to-point motions driven by a novel electromagnetic linear actuator, Mechatronics 23 (4) (2013) 445-451.

[39] J. Arcos-Legarda, A. Tovar, J. Cortes, H. Diaz, L. Sarmiento, Multivariable gpi control of a gait exoskeleton for people with walking disabilities, in: II International Congress of Engineering Mechatronics and Automation (CIIMA), 2013.

[40] N. Martínez-Fonseca, L. Á. Castañeda, A. Uranga, A. Luviano-Juárez, I. Chairez, Robust disturbance rejection control of a biped robotic system using high-order extended state observer, ISA transactions 62 (2016) 276-286.

[41] S. Tzafestas, M. Raibert, C. Tzafestas, Robust sliding-mode control applied to a 5-link biped robot, Journal of Intelligent and Robotic Systems 15 (1) (1996) 67-133.

[42] Y. Hurmuzlu, D. B. Marghitu, Rigid body collisions of planar kinematic chains with multiple contact points, The international journal of robotics research 13 (1) (1994) 82-92.

[43] J. W. Grizzle, G. Abba, F. Plestan, Asymptotically stable walking for biped robots: Analysis via systems with impulse effects, Automatic Control, IEEE Transactions on 46 (1) (2001) 51-64.

[44] H. Sira-Ramirez, F. Beltrán-Carbajal, A. Blanco-Ortega, A generalized proportional integral output feedback controller for the robust perturbation rejection in a mechanical system, STA 5 (4) (2008) 24-32.

[45] H. Sira-Ramírez, C. G. Rodríguez, J. C. Romero, A. L. Juárez, Algebraic identification and estimation methods in feedback control systems, John Wiley \& Sons, 2014.

[46] H. Sira-Ramírez, A. Luviano-Juárez, M. Ramírez-Neria, R. GarridoMoctezuma, Flat filtering: A classical approach to robust control of nonlinear systems, in: American Control Conference (ACC), 2016, IEEE, 2016, pp. 3844-3849. 
[47] J. Cortés-Romero, G. A. Ramos, H. Coral-Enriquez, Generalized proportional integral control for periodic signals under active disturbance rejection approach, ISA transactions 53 (6) (2014) 1901-1909.

[48] S. Veer, Rakesh, I. Poulakakis, Poincaré analysis for hybrid periodic orbits of systems with impulse effects under external inputs, CoRR abs/1712.03291. arXiv:1712.03291. URL http://arxiv.org/abs/1712.03291

[49] C. Canudas-de Wit, B. Espiau, C. Urrea, Orbital stabilization of underactuated mechanical systems, IFAC Proceedings Volumes 35 (1) (2002) $527-532$.

[50] J. W. Grizzle, C. Chevallereau, Virtual constraints and hybrid zero dynamics for realizing underactuated bipedal locomotion, arXiv preprint arXiv:1706.01127.

[51] J.-J. E. Slotine, W. Li, et al., Applied nonlinear control, no. 1, Prentice hall Englewood Cliffs, NJ, 1991.

[52] Y. C. Kim, L. H. Keel, S. P. Bhattacharyya, Transient response control via characteristic ratio assignment, IEEE Transactions on Automatic Control 48 (12) (2003) 2238-2244.

[53] S. Veer, M. S. Motahar, I. Poulakakis, On the adaptation of dynamic walking to persistent external forcing using hybrid zero dynamics control, in: Intelligent Robots and Systems (IROS), 2015 IEEE/RSJ International Conference on, IEEE, 2015, pp. 997-1003.

[54] S. Collins, A. Ruina, R. Tedrake, M. Wisse, Efficient bipedal robots based on passive-dynamic walkers, Science 307 (5712) (2005) 1082-1085.

[55] E. R. Westervelt, Toward a coherent framework for the control of planar biped locomotion, Ph.D. thesis, University of Michigan Ann Arbor, MI (2003).

[56] K. Sreenath, Feedback control of a bipedal walker and runner with compliance., Ph.D. thesis, University of Michigan Ann Arbor, MI (2011). 


\section{Appendix A. Derivation of the HZDU}

In order to describe the internal dynamics, a transformation of the last row of the decomposed model (15) is developed. This part of the model is directly related to the acceleration $\ddot{q}_{N}$. First, let us consider the Lagrange differential equation used to find the underactuated part of the robot model,

$$
\frac{d}{d t} \frac{\partial \mathcal{L}}{\partial \dot{q}_{N}}-\frac{\partial \mathcal{L}}{\partial q_{N}}=\tilde{\gamma}_{\xi 2}\left(q_{s}, \dot{q}_{s}\right),
$$

which has a control input equal to zero and is affected by the model uncertainties and external disturbances $\tilde{\gamma}_{\xi 2}\left(q_{s}, \dot{q}_{s}\right)$. Using the kinetic energy $\mathcal{K}\left(q_{s}, \dot{q}_{s}\right)$ and the potential energy $\mathcal{V}\left(q_{s}\right)$, the Lagrangian is expressed as

$$
\mathcal{L}:=\mathcal{K}\left(q_{s}, \dot{q}_{s}\right)-\mathcal{V}\left(q_{s}\right),
$$

and the Lagrange differential equation A.1 can be written as

$$
\begin{gathered}
\frac{d}{d t}\left(\frac{\partial \mathcal{K}\left(q_{s}, \dot{q}_{s}\right)}{\partial \dot{q}_{N}}\right)-\frac{d}{d t}\left(\frac{\partial \mathcal{V}\left(q_{s}\right)}{\partial \dot{q}_{N}}\right)+\cdots \\
\cdots-\frac{\partial \mathcal{K}\left(q_{s}, \dot{q}_{s}\right)}{\partial q_{N}}+\frac{\partial \mathcal{V}\left(q_{s}\right)}{\partial q_{N}}=\tilde{\gamma}_{\xi 2}\left(q_{s}, \dot{q}_{s}\right) .
\end{gathered}
$$

Following the concept that the kinetic energy is independent of the world frame and that the angle $q_{N}$ determines the orientation of the robot with respect to that frame, then

$$
\frac{\partial \mathcal{K}\left(q_{s}, \dot{q}_{s}\right)}{\partial q_{N}}=0 .
$$

In addition, since the potential energy is not a function of the velocity, then

$$
\frac{\partial \mathcal{V}\left(q_{s}\right)}{\partial \dot{q}_{N}}=0 .
$$

Thus, (A.3) takes the form

$$
\frac{d}{d t}\left(\frac{\partial \mathcal{K}\left(q_{s}, \dot{q}_{s}\right)}{\partial \dot{q}_{N}}\right)+\frac{\partial \mathcal{V}\left(q_{s}\right)}{\partial q_{N}}=\tilde{\gamma}_{\xi 2}\left(q_{s}, \dot{q}_{s}\right) .
$$

Considering that

$$
\mathcal{K}\left(q_{s}, \dot{q}_{s}\right)=\frac{1}{2} \dot{q}_{s}^{\mathrm{T}} D\left(q_{s}\right) \dot{q}_{s},
$$


then, $\partial \mathcal{K}\left(q_{s}, \dot{q}_{s}\right) / \partial \dot{q}_{N}$ can be simplified through the following procedure:

$$
\begin{aligned}
& \frac{\partial \mathcal{K}\left(q_{s}, \dot{q}_{s}\right)}{\partial \dot{q}_{N}}=\frac{\partial}{\partial \dot{q}_{N}}\left(\frac{1}{2} \dot{q}_{s}^{\mathrm{T}} D\left(q_{s}\right) \dot{q}_{s}\right), \\
& =\frac{1}{2} \frac{\partial}{\partial \dot{q}_{N}}\left(\left[\begin{array}{ll}
\dot{q}_{b} & \dot{q}_{N}
\end{array}\right]\left[\begin{array}{ll}
D_{11}\left(q_{s}\right) & D_{12}\left(q_{s}\right) \\
D_{21}\left(q_{s}\right) & D_{22}\left(q_{s}\right)
\end{array}\right]\left[\begin{array}{c}
\dot{q}_{b} \\
\dot{q}_{N}
\end{array}\right]\right), \\
& =\left[\begin{array}{ll}
D_{21}\left(q_{s}\right) & D_{22}\left(q_{s}\right)
\end{array}\right]\left[\begin{array}{c}
\dot{q}_{b} \\
\dot{q}_{N}
\end{array}\right], \\
& =D_{n}\left(q_{s}\right) \dot{q}_{s} .
\end{aligned}
$$

Replacing A.8 into A.6 yields

$$
\frac{d}{d t}\left(D_{n}\left(q_{s}\right) \dot{q}_{s}\right)+\frac{\partial \mathcal{V}\left(q_{s}\right)}{\partial q_{N}}=\tilde{\gamma}_{\xi 2}\left(q_{s}, \dot{q}_{s}\right) .
$$

In A.9, the internal dynamic states can be defined as

$$
\begin{aligned}
& \eta_{\xi 1}:=D_{n}\left(q_{s}\right) \dot{q}_{s}, \\
& \eta_{\xi 2}:=\Theta\left(q_{s}\right),
\end{aligned}
$$

where $\Theta\left(q_{s}\right)$ is a function of $q_{N}$ that represents the angle between the ground and the virtual link that connects the support leg-end with the hip as shown in Figure 1. Hence, the underactuated dynamics of the system can be described by

$$
\begin{aligned}
& \dot{\eta}_{\xi 1}=\frac{d}{d t}\left(D_{n}\left(q_{s}\right) \dot{q}_{s}\right)=\tilde{\gamma}_{\xi 2}\left(q_{s}, \dot{q}_{s}\right)-\frac{\partial V\left(q_{s}\right)}{\partial q_{N}} \\
& \dot{\eta}_{\xi 2}=\frac{\partial \Theta\left(q_{s}\right)}{\partial q_{s}} \dot{q}_{s} .
\end{aligned}
$$

If there is an initial condition $q_{s}\left(t_{0}\right)$ for which $e_{q_{i}}\left(t_{0}\right) \approx 0$ and a control signal $u^{*}$ such that $e_{q_{i}}(t) \approx 0$, for all $t>t_{0}$, where $t_{0}$ represents the initial time, then the system is constrained by the conditions $q_{b}=q_{d}+e_{q}$ and $\dot{q}_{b}=\dot{q}_{d}+\dot{e}_{q}$. Therefore,

$$
\left.\frac{d e_{q_{i}}}{d t}\right|_{\substack{q_{b}=q_{d}+e_{q} \\ \dot{q}_{b}=\dot{q}_{d}+\dot{e}_{q}}}=\left.\frac{\partial e_{q_{i}}}{\partial q_{s}} \dot{q}_{s}\right|_{\substack{q_{b}=q_{d}+e_{q} \\ \dot{q}_{b}=\dot{q}_{d}+\dot{e}_{q}}}=0 .
$$


Using (A.10) and A.14, the angular velocity vector can be expressed as

$$
\dot{q}_{s}=\left[\begin{array}{c}
\frac{\partial e_{q_{i}}}{\partial q_{s}} \\
D_{n}\left(q_{s}\right)
\end{array}\right]^{-1}\left[\begin{array}{c}
0_{4 \times 1} \\
I_{1 \times 1}
\end{array}\right] \eta_{\xi 1} \mid \begin{gathered}
\\
\begin{array}{c}
q_{b}=q_{d}+e_{q} \\
\dot{q}_{b}=\dot{q}_{d}+\dot{e}_{q}
\end{array}
\end{gathered},
$$

and (A.12) and (A.13) take the form

$$
\begin{aligned}
& \dot{\eta}_{\xi 1}=\left.\left(\tilde{\gamma}_{\xi 2}\left(q_{s}, \dot{q}_{s}\right)-\frac{\partial \mathcal{V}\left(q_{s}\right)}{\partial q_{N}}\right)\right|_{\begin{array}{l}
q_{b}=q_{d}+e_{q} \\
\dot{q}_{b}=\dot{q}_{d}+\dot{e}_{q}
\end{array}}, \\
& \dot{\eta}_{\xi 2}=\frac{\partial \Theta\left(q_{s}\right)}{\partial q_{s}}\left[\begin{array}{c}
\frac{\partial e_{q_{j}}}{\partial q_{s}} \\
D_{n}\left(q_{s}\right)
\end{array}\right]^{-1}\left[\begin{array}{c}
0_{4 \times 1} \\
I_{1 \times 1}
\end{array}\right] \eta_{\xi 1} \mid \begin{array}{l}
\eta_{q_{b}=q_{d}+e_{q}} \\
\dot{q}_{b}=\dot{q}_{d}+\dot{e}_{q}
\end{array}
\end{aligned} .
$$

Finally, the zero dynamics with uncertainties can be expressed as the sum of a nominal zero dynamics without uncertainties and two signals that lump the uncertainties and disturbances in the internal dynamics with the effect of small tracking errors in the controlled joints. This is,

$$
\begin{aligned}
& \dot{\eta}_{\xi 1}=\tilde{\kappa}_{1}+\gamma_{\eta 1}, \\
& \dot{\eta}_{\xi 2}=\tilde{\kappa}_{2} \eta_{\xi 1}+\gamma_{\eta 2},
\end{aligned}
$$

where

$$
\begin{aligned}
& \tilde{\kappa}_{\xi 1}=-\left.\frac{\partial \mathcal{V}\left(q_{s}\right)}{\partial q_{5}}\right|_{q_{b}=q_{d}, \dot{q}_{b}=\dot{q}_{d}}, \\
& \tilde{\kappa}_{\xi 2}=\left.\frac{\partial \Theta\left(q_{s}\right)}{\partial q_{s}}\left[\begin{array}{c}
\frac{\partial q_{j}}{\partial q_{s}} \\
D_{n}\left(q_{s}\right)
\end{array}\right]^{-1}\left[\begin{array}{c}
0_{4 \times 1} \\
I_{1 \times 1}
\end{array}\right]\right|_{q_{b}=q_{d}, \dot{q}_{b}=\dot{q}_{d}},
\end{aligned}
$$

$\gamma_{\eta 1}$ and $\gamma_{\eta 2}$ are the lumped disturbance signals in the internal dynamics of the robot. In compact form, (A.18) and (A.19) are written as

$$
\dot{z}=f_{z}(z)+\Xi,
$$

where

$$
z=\left[\begin{array}{c}
\eta_{\xi 1} \\
\eta_{\xi 2}
\end{array}\right], \quad f_{z}(z)=\left[\begin{array}{c}
\tilde{\kappa}_{1} \\
\tilde{\kappa}_{2} \eta_{\xi 1}
\end{array}\right], \quad \Xi=\left[\begin{array}{c}
\gamma_{\eta 1} \\
\gamma_{\eta 2}
\end{array}\right] .
$$


The zero dynamics states are exposed to the discrete event of the supportleg exchange. This natural walking feature implies that the zero dynamics has a hybrid behavior. Therefore, the zero dynamics state vectors before and after the impact are described by $z_{\xi}^{-}=\left[\begin{array}{ll}\eta_{\xi 1}^{-} & \eta_{\xi 2}^{-}\end{array}\right]^{\mathrm{T}}$ and $z_{\xi}^{+}=\left[\begin{array}{ll}\eta_{\xi 1}^{+} & \eta_{\xi 2}^{+}\end{array}\right]^{\mathrm{T}}$, respectively. The zero dynamics state vector after the impact is defined by

$$
\begin{aligned}
& \eta_{\xi 1}^{+}=D_{n}\left(q_{s}^{+}\right) \dot{q}_{s}^{+}, \\
& \eta_{\xi 2}^{+}=\Theta\left(q_{s}^{+}\right) .
\end{aligned}
$$

Using (7) the post-impact states A.24 and A.25 can be expressed in a compact form as

$$
z^{+}=\Delta_{z}\left(z^{-}\right)+\Xi_{\Delta} .
$$

With the zero dynamics A.23 and the reset law A.26), the resulting hybrid zero dynamics with uncertainties (HZDU) can be expressed as

$$
\Sigma_{z}: \begin{cases}\dot{z}=f_{z}(z)+\Xi, & z^{-} \notin \mathcal{Z}, \\ z^{+}=\Delta_{z}\left(z^{-}\right)+\Xi_{\Delta}, & z^{-} \in \mathcal{Z} .\end{cases}
$$

where $\mathcal{Z} \subset \mathcal{S}$ and it is defined as

$$
\mathcal{Z}:=\left\{\left[\begin{array}{ll}
q_{s}^{\mathrm{T}} & \dot{q}_{s}^{\mathrm{T}}
\end{array}\right]^{\mathrm{T}} \in \mathcal{S} \mid q_{b}=q_{d}, \dot{q}_{b}=\dot{q}_{d}\right\} .
$$

